\title{
ADAM: A Realistic Implementation for a W-CDMA Smart Antenna
}

\author{
Ramón Martínez Rodríguez-Osorio \\ Department of Signals, Systems and Radiocommunications, Polytechnic University of Madrid, 28040 Madrid, Spain \\ Email: ramon@gr.ssr.upm.es
}

Laura García García

Department of Signals, Systems and Radiocommunications, Polytechnic University of Madrid, 28040 Madrid, Spain Email:lgg@gr.ssr.upm.es

\begin{abstract}
Alberto Martínez Ollero
Department of Signals, Systems and Radiocommunications, Polytechnic University of Madrid, 28040 Madrid, Spain Email: alberto@gr.ssr.upm.es
\end{abstract}

\section{Francisco Javier García-Madrid Velázquez}

Department of Signals, Systems and Radiocommunications, Polytechnic University of Madrid, 28040 Madrid, Spain Email: javiergmv@gr.ssr.upm.es

\section{Leandro de Haro Ariet}

Department of Signals, Systems and Radiocommunications, Polytechnic University of Madrid, 28040 Madrid, Spain Email: leandro@gr.ssr.upm.es

\section{Miguel Calvo Ramón}

Department of Signals, Systems and Radiocommunications, Polytechnic University of Madrid, 28040 Madrid, Spain Email:miguel@gr.ssr.upm.es

Received 30 May 2003; Revised 28 November 2003

\begin{abstract}
Adaptive-type smart antennas do not usually operate on the deployed universal mobile telecommunication system (UMTS) scenarios, although UTRA (UMTS terrestrial radio access) foresees their operation and they would improve capacity especially in mixed-service environments. This paper describes the implementation of a software radio-based version of an adaptive antenna, named ADAM, that can be used with any standard Node B, both in the up- and downlinks. This transparent operational feature has been made possible by the partial cancelation algorithm applied in the uplink by means of a common beamforming vector. Firstly, a general description of the system as well as the theory of its operation are described. Next, the hardware architecture is presented, showing the real implementation. Also a complete software description is done. Finally, results are presented, obtained from both simulation and real implementation, showing the improvement obtained with the adaptive antenna as compared with a typical sectored one. Performance results obtained in the initial tests show that ADAM prototype provides an SINR increase of 12.5 and $6.5 \mathrm{~dB}$ over a conventional sectored antenna in the uplink and downlink, respectively. System-level simulation results are presented, showing the throughput increase obtained with ADAM. These findings provide evidence of the capacity improvement achieved with the ADAM prototype.
\end{abstract}

Keywords and phrases: smart antenna prototype, beamforming, wireless communications, synchronization, DSP, UMTS.

\section{INTRODUCTION}

The smart antenna concept is applied to several kinds of antenna arrays. Phased arrays, switched multibeam antennas, and adaptive array antennas are usually included under the smart antenna concept with the only condition of includ- ing the possibility to somehow control the radiation pattern. Great advantages have been reported for the smart antenna implementation in base stations for mobile telephone communications, but this kind of antenna has not been extensively applied to those systems yet. 
If capabilities of phased array, switched-beam array, and adaptive array antennas are compared, the last type shows considerable advantages over the others [1]. Not only can adaptive arrays improve antenna gain in the user direction but they can also cancel interferences inside the angular range of control. This ability implies an increase of the signalto-interference-plus-noise ratio (SINR) for each user. For code division multiple access (CDMA) systems, an increase of sector capacity is obtained for those cells with base stations equipped with smart antennas. The capacity increase is higher in cells with high interference levels, usually produced by high bit rate users.

Adaptive antenna systems can be implemented using a space or time reference-based algorithm. In spatial reference adaptive arrays, interference directions are computed and the array weights are obtained to cancel or minimize them. In time reference adaptive arrays, time series from the input signal at each array element are processed to form the array vector of weights. The array factor implemented for each user increases the SINR and improves the energy per bit to noise density ratio $\left(\mathrm{E}_{b} / \mathrm{N}_{0}\right)$ due to the correlation of the received signals. This strategy is appropriate for CDMA signals since a time reference can be obtained applying the user code. In the particular case of universal mobile telecommunication system (UMTS), the physical layer has been designed to work with adaptive antennas both in uplink and downlink [2].

A significant research effort has taken place in the last years to introduce smart antenna systems in cellular scenarios. However, the deployment of these antenna systems has not become a reality yet due to their cost and complexity. In practice, only switched-beam antennas for second generation $(2 \mathrm{G})$ systems have been commercially deployed $[3,4,5,6,7,8]$. This is due to the complexity of adaptive antennas in third generation $(3 \mathrm{G})$ systems. In contrast to $2 \mathrm{G}$ systems, where beamforming can be done in radio frequency (RF), beamforming in 3G must be applied after demodulating the CDMA signal so that adaptive antenna functions need to be integrated into the (digital and intermediate frequency (IF)) baseband-processing sections of the base station. Therefore, the implementation of adaptive antennas in $3 \mathrm{G}$ base stations requires a reconfigurable and flexible architecture. These features can be obtained using software radio platforms $[9,10,11]$.

Many of the existing smart antenna solutions for $3 \mathrm{G}$ have been developed for a unique base station equipment manufacturer $[12,13]$. This fact makes the deployment of smart antenna systems unfeasible for mobile communications operators due to the high associated cost and manufacturer dependency. A plug and play smart antenna solution, appropriate for any base station from any manufacturer, has not been developed yet.

This paper details a practical implementation of an adaptive plug and play smart antenna for $3 \mathrm{G}$ mobile communication systems based on wideband-CDMA (W-CDMA) like UMTS $[14,15]$. Unlike currently existing adaptive antenna arrays, the implementation described here implies an easy deployment over any base station, not only on those specifically developed to be used with smart antennas [16]. ADAM stands for "adaptive antenna for multioperator scenarios," as it can be connected to any base station site even shared by several operators.

As a plug and play functionality is demanded, the UMTS signals are demodulated and remodulated again, allowing a direct connection between the smart antenna outputs and the base station inputs [16]. Due to this process, in the uplink, only those interferences common to the intracellular users and all the extracellular interferences are canceled. The relationship between the extracellular and intracellular interferences is called the extracellular interference factor $F$ and has a value between 0.4 to 1.4 depending on the environment and the service [15]. This implies that more than $50 \%$ of the interferences are canceled on average as the common intracellular interferences should also be taken into account.

This antenna will take profit of hot spots, improving the capacity in the vicinity of high occupied cells. In these situations, mainly higher power external interferences from multimedia services are canceled by ADAM prototype, as it is demonstrated by simulation in this paper. In these situations, the antenna would help the cells in the vicinity of a hot spot to expand their coverage and to compensate the "cell breathing" of high occupied cells. Moreover, in mixed and asymmetric services scenarios, typical of $3 \mathrm{G}$ systems, ADAM will increase the capacity in terms of total throughput.

According to the software radio concept, the analog-todigital conventers (ADCs) and digital-to-analog conventers (DACs) are located just before the analog RF-to-IF chains, hence working with IF signals instead of the typical baseband signal. This allows most of the system modules to be implemented in software, which is a great advantage with respect to pure hardware implementations because the system can be easily reconfigured and updated with more advanced versions. Therefore, a great flexibility is achieved with this structure.

The beamforming module has been implemented just before the W-CDMA modulation. In the uplink, classical beamforming algorithms have been adapted to the special extracellular cancelation scheme implemented $[17,18]$. Although different beamforming algorithms can be used, the normalized least mean squares (NLMS) algorithm has been selected initially due to its reduced computational complexity. In the downlink, beamforming aims to cancel all intraand extracellular interferences, thus a full cancelation algorithm has been selected.

Apart from NLMS, some tests have been done using the recursive least squares (RLS) algorithm in order to study the performance improvement obtained in the convergence speed and final SINR.

It is important to remark on the implementation of the synchronization algorithms in UMTS $[19,20,21]$. This problem has been solved using a two-step approach, initially doing a coarse synchronization that is followed by a continuous fine synchronization. The implemented algorithm has been intensively optimised.

As the smart antenna should be transparent for the base station, it should not implement the base stations physical procedures, such as power control and handover, which are 


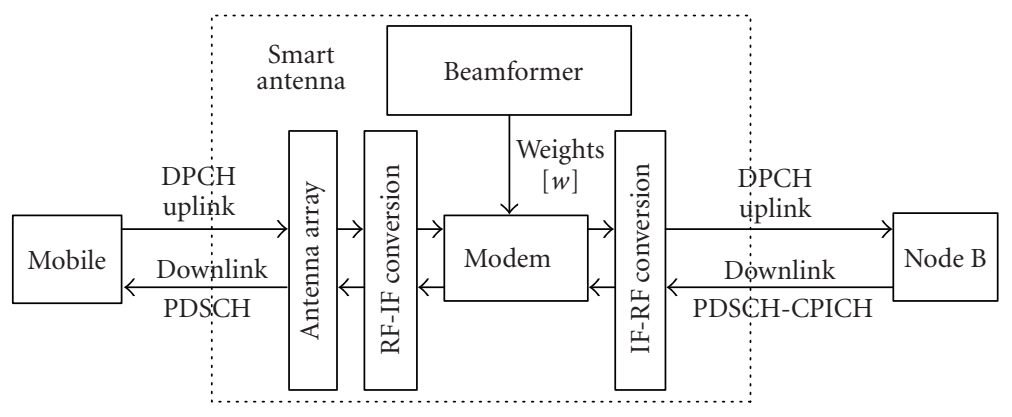

FIGURE 1: Implementation architecture of the ADAM smart antenna to be deployed in connection with a standard Node B.

performed by the base station (Node B) itself. Moreover, polarization diversity is performed by the base station, and the ADAM antenna is connected to both base station ports and processes each polarization independently.

\section{UMTS SMART ANTENNA ARCHITECTURE AND OPERATION OF ADAM PROTOTYPE}

The implemented architecture of the ADAM smart antenna prototype is shown in Figure 1. In the downlink, the RF signal from Node B is downconverted to IF, digitized, demodulated, beamformed (with a set of different weights for each user), and finally, upconverted to RF. In the uplink, an equivalent process is performed but using a common beamforming vector for all the users. This architecture performs a total interference cancelation in the downlink but only a partial cancelation in the uplink.

However, a higher flexibility is achieved because ADAM antenna can be plugged to any base station, even those not especially designed to work with a smart antenna system [16]. All the commercial Nodes B have a standardized RF interface ( $U u$ interface). In case of using a baseband interface for the connection of the smart antenna with the base station, the interface definition would depend on each particular manufacturer, and ADAM prototype would lose its transparent operation feature. Therefore, once the array output has been computed, it must be upconverted again to the original RF carrier in order to interface adequately with any standard Node B, as it can be seen in Figure 1.

According to the physical layer of UMTS, time reference and user synchronization may be obtained in the uplink from the dedicated channel (DCH) (in particular, dedicated physical control channel (DPCCH)) [17]. However, downlink allows several ways to obtain time reference and user synchronization: common pilot channel $(\mathrm{CPICH})$, primary common control physical channel (P-CCPCH), secondary$\mathrm{CCPCH}$ (S-CCPCH), and even pilot symbols or diversity pilots [15]. ADAM implementation gets user synchronization from DPCCH in the uplink, and from CPICH in the downlink. Tables 1 and 2 summarize which physical channels are processed in up and down streams to get system information and which channels are beamformed or not by the ADAM prototype.

In the uplink, both common and dedicated channels are beamformed since the beamformer for dedicated channels
TABLE 1: Beamforming of uplink physical channels. (PRACH: physical random access channel.)

\begin{tabular}{lcc}
\hline Channel & Function in smart antenna & Beamforming \\
\hline DPCCH & $\begin{array}{c}\text { User synchronization and uplink } \\
\text { channel characterization }\end{array}$ & Yes \\
DPDCH & - & Yes \\
PRACH & - & Yes \\
\hline
\end{tabular}

TABLE 2: Beamforming of downlink physical channels. (AICH: acquisition indicator channel; $\mathrm{CSICH}$ : common packet channel status indicator channel; PICH: page indication channel; PDSCH: physical downlink shared channel.)

\begin{tabular}{lcc}
\hline Channel & Function in smart antenna & Beamforming \\
\hline SCH & Cell slot synchronization & No \\
DPICH & $\begin{array}{c}\text { Downlink frame synchronization } \\
\text { User synchronization }\end{array}$ & No \\
(scrambling code identification) & \\
P-CCPCH & - & No \\
S-CCPCH & - & No \\
AICH & - & No \\
CSICH & - & No \\
PICH & - & No \\
DPCH & - & Yes \\
PDSCH $(\mathrm{DPCH})$ & - & Yes \\
\hline
\end{tabular}

adapts simultaneously the common channels coming from the users directions. Figure 2 shows the proposed architecture for the uplink, where the DPCCH from each user is synchronized and demodulated to perform the computation of individual beamforming weights. At this stage, the common set of weights are computed and applied to the composite received UMTS signal.

In the downlink, common and broadcast channels are bypassed and transmitted to the whole sector in parallel with the beamformed dedicated channels, as it can be seen in Figure 3. The synchronization is performed using primary$\mathrm{CPICH}$ (P-CPICH) information and applied to every user to be demodulated, beamformed, and remodulated again before being sent to each antenna element. Downlink weights are obtained from uplink weights, as it will be explained in Section 4.2.

The performance improvement that may be achieved with an adaptive antenna depends on the following aspects: 


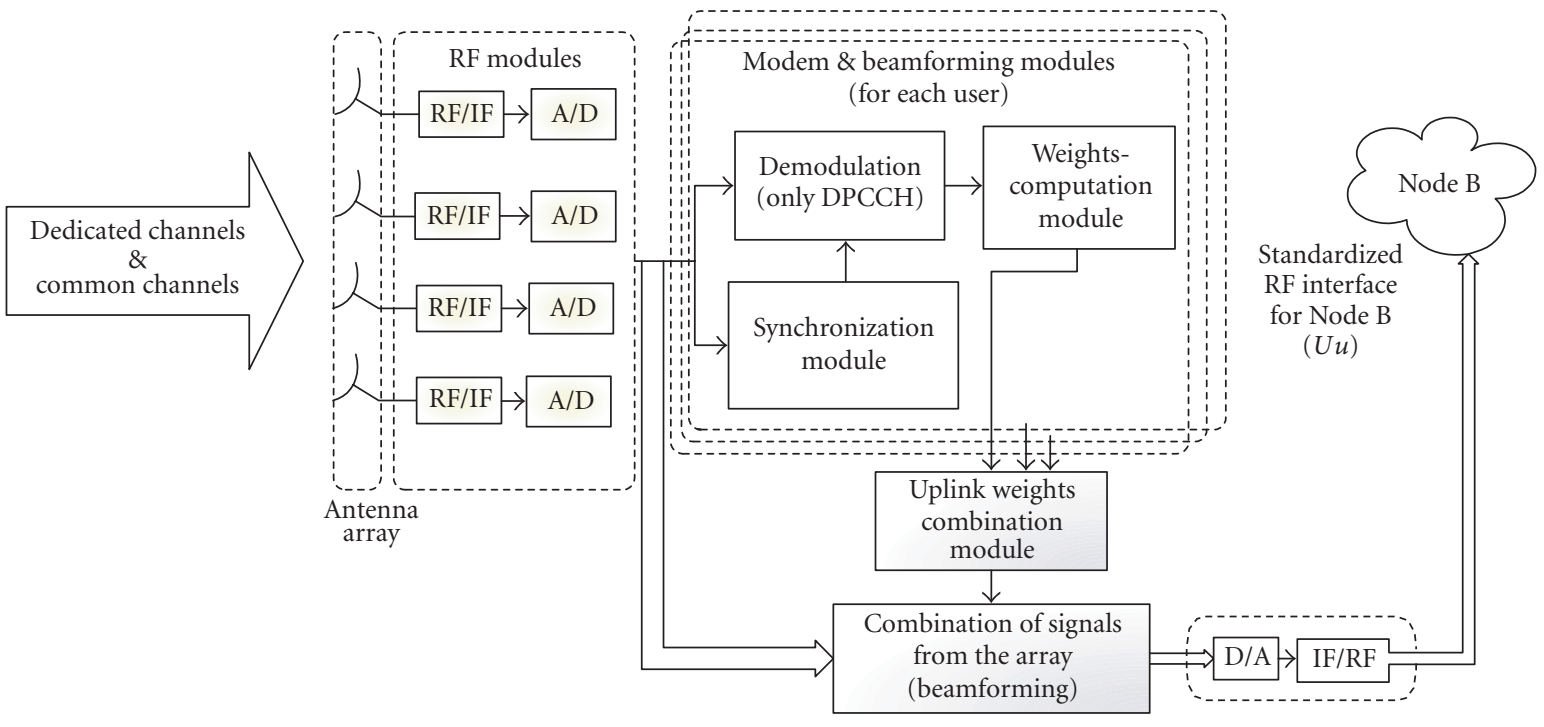

FIGURE 2: Uplink block diagram (1 polarization).

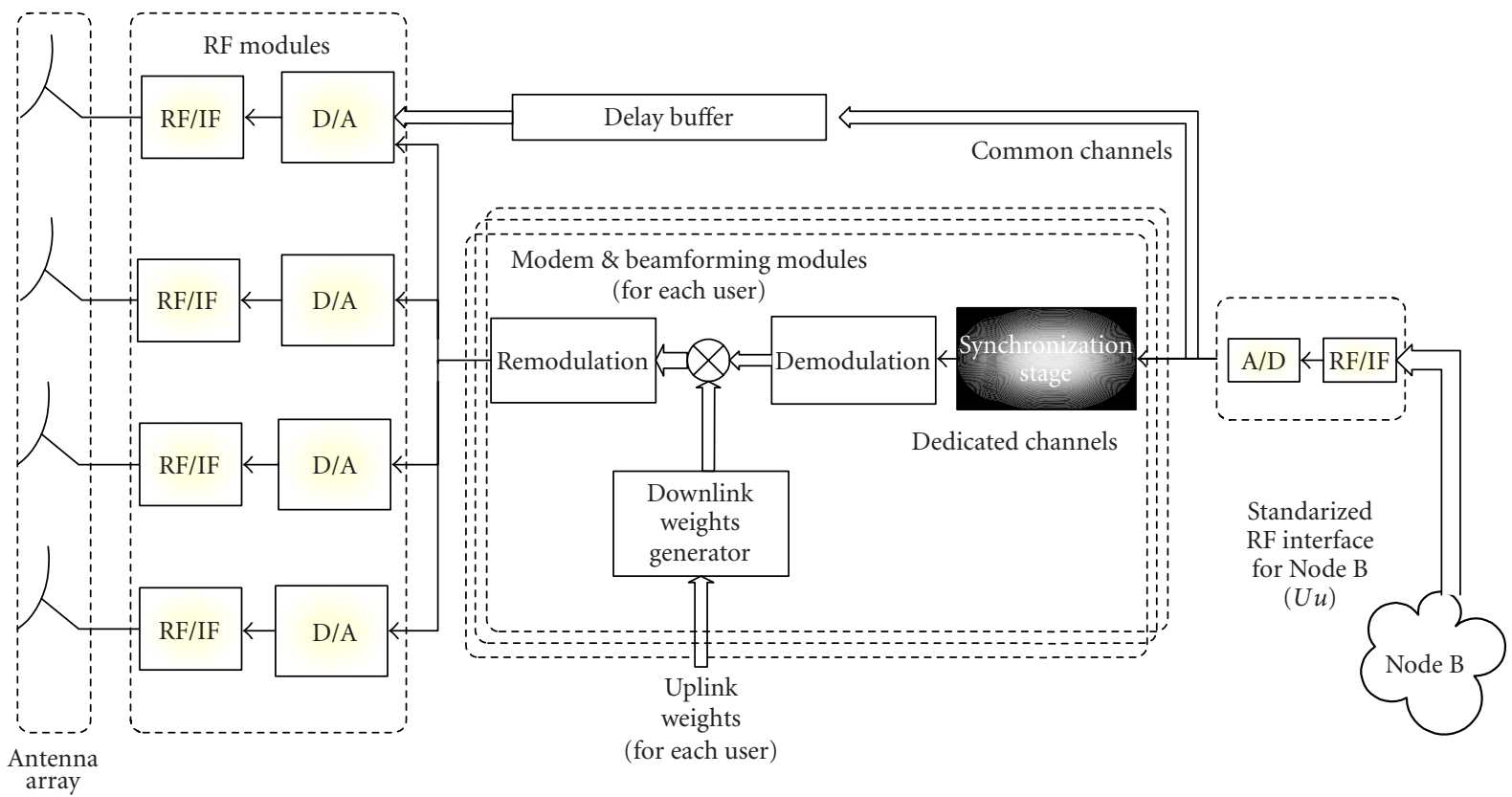

FIGURE 3: Downlink block diagram (1 polarization).

antenna array geometry, adaptive algorithm that controls the beamforming process, and propagation and interference environment. Those issues have been studied by simulation and are presented in Section 6. The ADAM array prototype uses four commercial sectored antennas for the UMTS band, each with a $-3 \mathrm{~dB}$ beamwidth of $65^{\circ}$ and $\pm 45^{\circ}$ polarization ports [22]. The individual antennas are put together in a uniform linear array structure, as shown in Figure 4 . With this configuration, interelement separation is $15 \mathrm{~cm}$ (wide dimension of each sectored antenna), which is equivalent to $0.975 \lambda$ and $1.070 \lambda$ at the uplink and downlink frequencies, respectively.

\section{HARDWARE ARCHITECTURE}

The overall system proposed in this paper is formed by several hardware devices. Their characteristics, as well as the final selected hardware architecture, are presented below for both the uplink and downlink. Although a general description of the adaptive antenna has been made in Section 2, we focus here on the specific selected hardware solutions.

In the uplink, the received analog signal is downconverted by the RF-to-IF chains and digitalized. Afterwards, it is processed in the digital signal processing module, where 


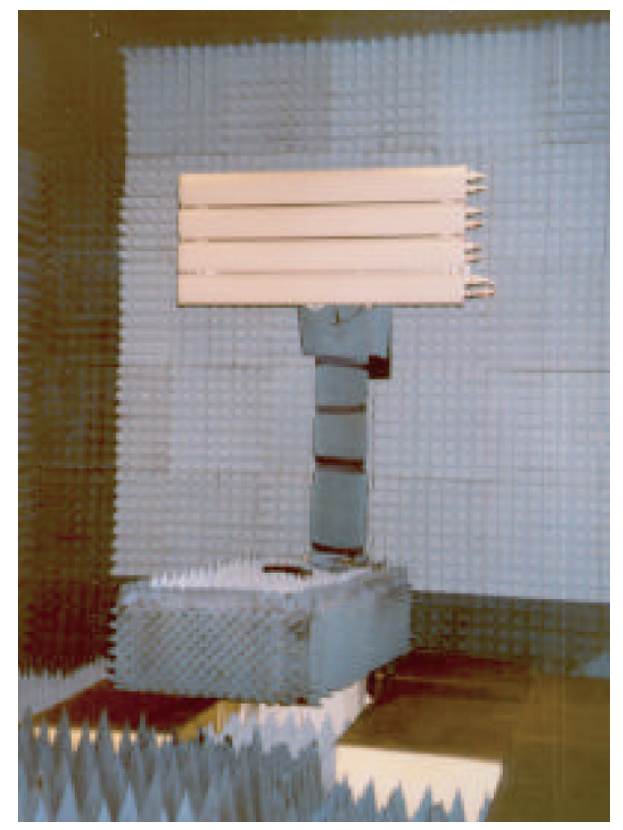

FIgURE 4: ADAM prototype: antenna array structure.

several digital signal processors (DSPs) work in parallel. The processed baseband signal is then analog-converted again, sent to an IF-to-RF chain and then to the Node-B RF input port. Conversely, the signal received from the Node-B output port follows similar steps in the downlink (RF-to-IF conversion, digitalization, digital processing, analog conversion, and RF upconversion), being finally transmitted through the antenna array.

Figure 5 shows a general architecture of the hardware implementation, where the blocks for the two polarizations are identical. The digital processing module, formed by several processors, is common to both polarizations. Analog RF-toIF and IF-to-RF chains are not thoroughly explained here since it is out of the scope of this paper, mainly focused on the digital signal processing stages. Figure $6 a$ shows the development system for software radio modules, whereas Figure 6b shows the test equipment.

Due to the software radio implementation, the IF frequency value offered to the rest of the modules must be carefully selected. A high IF would simplify the design of the analog chains, especially the filtering of the image frequency, but it would increment the processing capacity requirements. Also the current state of the art in ADCs and DACs should be taken into account since there is a tradeoff between the vertical resolution and sample frequency that can be achieved. With this in mind, an IF of $44 \mathrm{MHz}$ was selected as a compromise solution.

Several aspects were taken into consideration to properly select the ADCs and DACs. The first one was the vertical resolution (or number of bits in conversion) required for this application. The quantification noise is lower with a high vertical resolution, but the available maximum sampling frequency decreases as the number of bits in conver- sion are incremented. The recommended number of bits to use in a UMTS application is at least 12 [1]. As for the maximum sampling frequency $f_{s, \max }$, it should be high enough to correctly receive or transmit the desired signal without loss of information. Also related to the $f_{s, \max }$, we have to take into account the conversion bandwidth parameter. Finally, the dynamic range of the input voltage should be considered, especially in the analog chains design, to properly adjust its gain to the ADC input and DAC output levels.

After the ADC, the signal must be downconverted to baseband by means of an IQ demodulator. One possibility could be to implement it directly in a general DSP. But due to the high UMTS sampling rate, the required computational capacity to accomplish that operation would make the implementation unfeasible. Another interesting solution would be to use on-chip IQ demodulators or broadband downconverters, usually called front-ends. These devices can process the signal independently of the general DSPs, which can be used then to do the subsequent processing. The latter option has been chosen to implement the downconversion to baseband; so a general-purpose receiver has been selected from the commercially available devices. The selected receiver boards ${ }^{1}$ consist of two broadband IQ demodulators plus two ADCs so that two identical receiver channels per receiver board are available [23]. The vertical resolution for the ADCs is 12 bits, and its maximum sampling frequency is $80 \mathrm{MHz}$. The ADC sampling frequency must be carefully selected. It has to be a multiple of the UMTS baseband signal rate $3.84 \mathrm{Mchip} / \mathrm{s}$, multiplied by the number of samples per chip, which is $N_{\mathrm{spc}}=4$ in this prototype. Neither $15.36 \mathrm{MHz}$ nor $30.72 \mathrm{MHz}$ can be used as sampling frequencies since it would cause aliasing in the sampled signal. On the other side, the ADC features restrict the possible sampling frequency to a maximum of $100 \mathrm{MHz}$. Thus, $f_{s}=61.44 \mathrm{MHz}$ has been chosen. Since $f_{s}$ does not meet the Nyquist theorem $\left(f_{s}\right.$ is lower than $\left.2 \cdot \mathrm{IF}\right)$, the resulting signal is undersampled. This does not involve a loss of information because the signal is bandlimited to $5 \mathrm{MHz}$. A diagram of the main parts of one receiving channel is shown in Figure 7.

Similarly, an IQ modulator is required before each DAC. Also the front-end solution has been adopted here. The selected digital upconversion boards ${ }^{2}$ provide two identical and independent broadband channels [23]. The DAC accepts 12-bit digital signal as input, and its maximum sampling frequency is $200 \mathrm{MHz}$. A block diagram of one channel can be seen in Figure 8.

Once the signal has been digitally converted and IQ demodulated, it has to be processed by the synchronization and beamforming modules, which are implemented in generalpurpose digital processors. A few characteristics have been considered to select the DSPs that have been used to implement the software modules. The most important features are the arithmetic type, the clock rate and, in connection with

\footnotetext{
${ }^{1}$ Pentek 6235-board.

${ }^{2}$ Pentek 6229-board.
} 


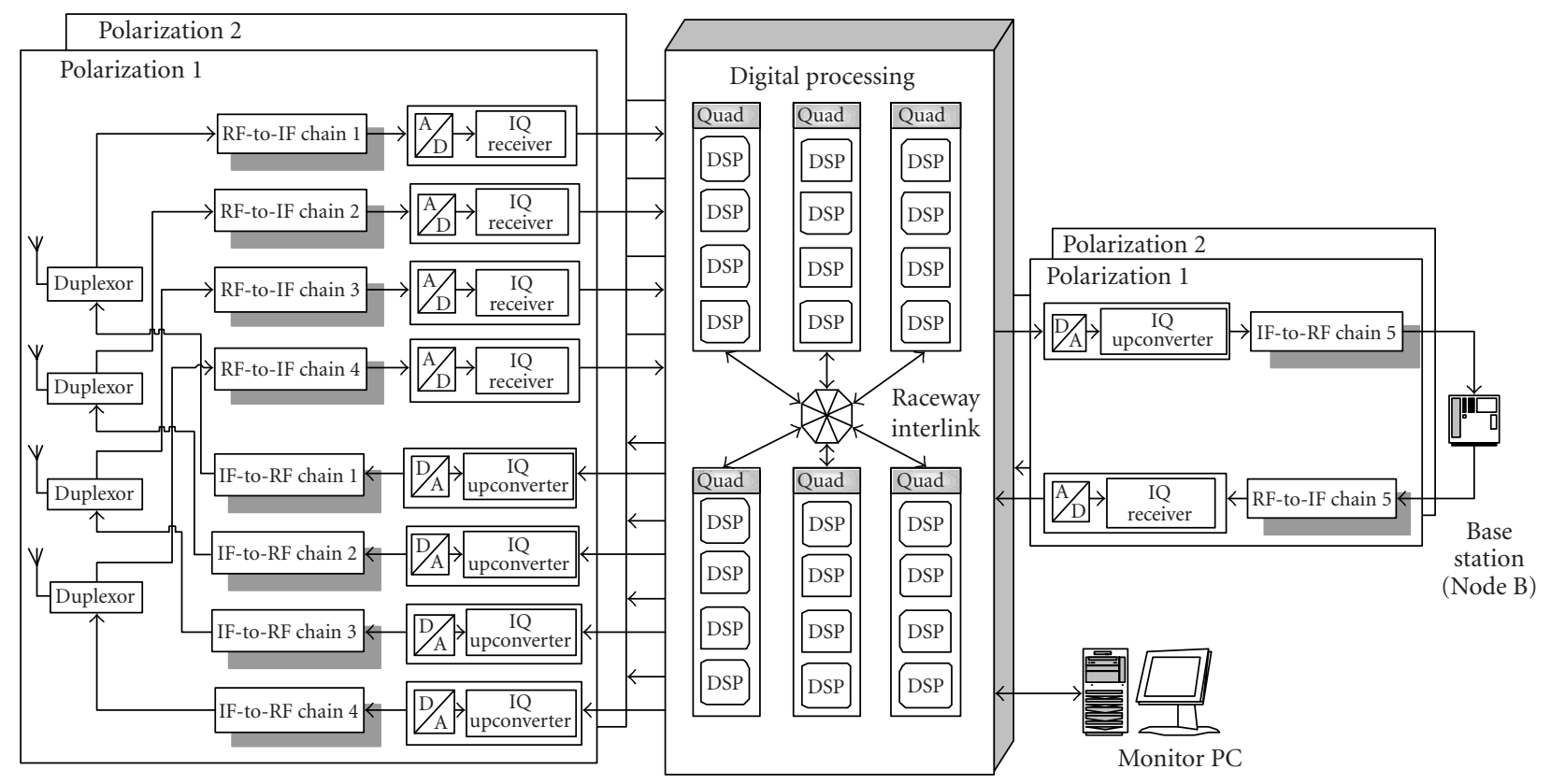

FIGURE 5: General hardware structure.

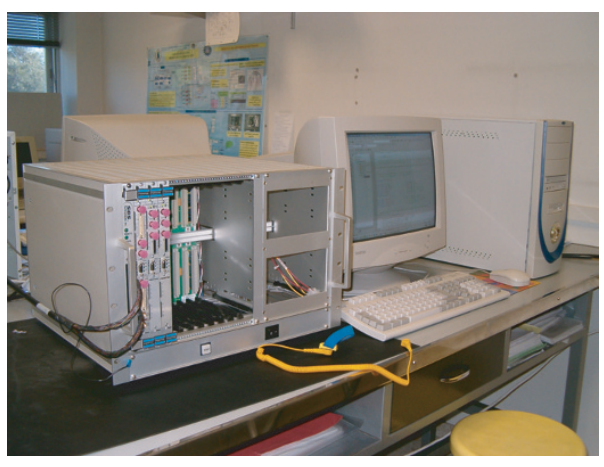

(a)

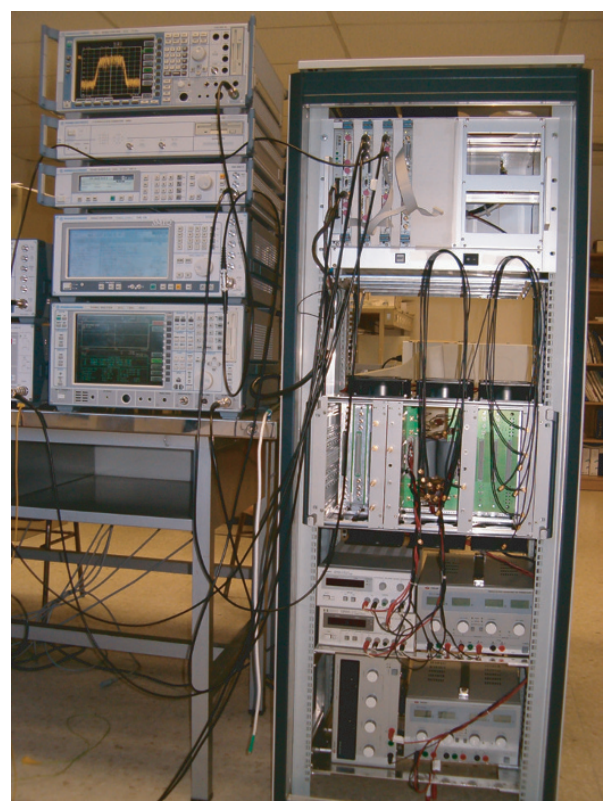

(b)

FIgURE 6: Hardware modules of ADAM prototype and test equipment. (a) Development system. (b) Measurement and test system.

this, the computational capacity. Fixed-point arithmetic is preferred instead of floating-point arithmetic since a higher speed processing for linear operations, like the ones required in this application, can be achieved. As regards the clock rate, the higher it is, the greater the number of instructions per second that can be executed, and the higher the computational capacity that can be obtained. In order to increase the computational capacity, a structure of various DSPs in paral- lel can be used. The selected digital processing structure consists of six 4-DSP boards, ${ }^{3}$ referred to as Quads [23]. Each Quad is formed by four $300-\mathrm{MHz}$ fixed-point DSPs along with other interfaces between DSPs. Every Quad is capable of

\footnotetext{
${ }^{3}$ Pentek 4292-Quad VME board, with four Texas instrument
} TMS30C6203 processors. 


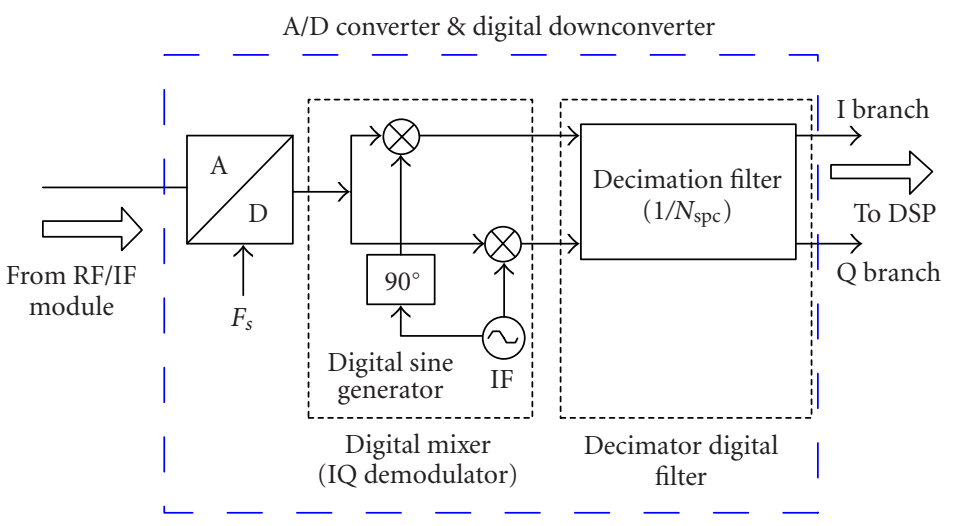

FIgURE 7: ADC and IQ demodulator.

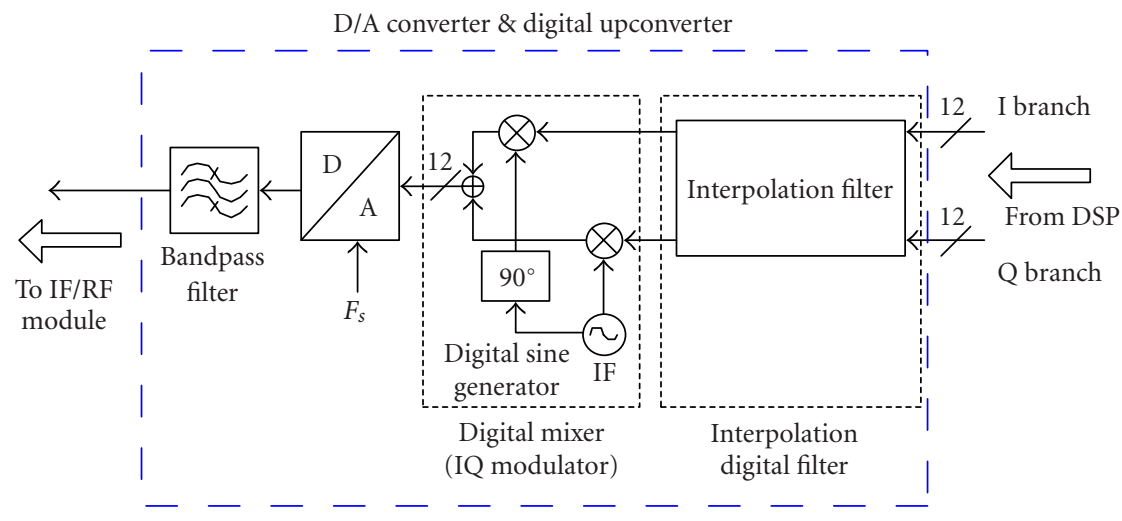

FIgURE 8: DAC and IQ modulator.

delivering a combined peak processing power of 9600 MIPS (millions of instructions per second).

In order to increase the data transfer rate between Quads, a high-speed data bus has been used ${ }^{4}[23,24]$. This device is a high-speed backplane fabric capable of delivering 32-bit word transfers between versa module eurocard (VME) boards, such as the Quads presented previously. It provides multiple, simultaneous high-speed communication paths between DSPs which make the bus a valuable asset to real-time applications. The bus is capable of communicating up to eight VME boards at a data transfer rate of $267 \mathrm{MBps}$, which means an aggregate transfer rate up to 1068 GBps.

For monitoring tasks, a personal computer can be connected to the digital processing module to control the process and allow viewing of key variables and parameters.

\section{PRINCIPLES AND IMPLEMENTATION OF SOFTWARE RADIO MODULES}

The software implementation has been divided into two main submodules: the set-up, synchronization and modem module, and the adaptive beamforming module. They are thoroughly explained below.

\footnotetext{
${ }^{4}$ Pentek 8251 Race++ interlink modules.
}

\subsection{Set-up, synchronization, and MODEM stages}

As it is known [2], each physical channel in W-CDMA is spread combining two types of codes with complementary properties: orthogonal variable spreading factor (OVSF) channelization codes and scrambling codes (Gold codes, with excellent correlation properties). Basic information needed in a W-CDMA process is the used codes and, like any spread-spectrum technique, the timing reference [25]. The function of the set-up stage is to find the essential data needed before the demodulation process in uplink and downlink.

\subsubsection{Set-up procedure}

Basic synchronization algorithms employed in the modem will be detailed in Section 4.1.2, and they are common for uplink and downlink. The main difference between uplink and downlink synchronization stages lies in which physical channels are used as reference signals.

In the downlink, all the physical channels (common signalling channels and dedicated user channels) use the same synchronization reference, that is, if the synchronization of one channel is known, the timing of the other channels is automatically known. The procedure to find the common timing reference for all downlink channels is called cell search procedure. Typically, cell search procedure is completed after three steps: slot synchronization, frame synchronization, 
TABLE 3: Number of clock cycles and acquisition time for the coarse synchronization algorithm.

\begin{tabular}{lccc}
\hline & Branches & Clock cycles/bit & Acquisition time (number of frames) \\
\hline Serial search & 1 & 3000 & 1 \\
\hline \multirow{4}{*}{ Parallel search } & 2 & 5700 & 0.5 \\
& $\mathbf{3}$ & $\mathbf{8 7 0 0}$ & $\mathbf{0 . 3 3}$ \\
& 4 & 10800 & 0.25 \\
& 10 & 27300 & 0.1 \\
\hline
\end{tabular}

code-group identification, and finally scrambling code identification [2]. Common signalling channels needed in this stage are the synchronization channel $(\mathrm{SCH})$ and the $\mathrm{P}$ $\mathrm{CPICH}$.

The first and second steps use SCH codes. During the first step, the cell slot synchronization is acquired; it can be done by correlating the received signal of the base station with the primary SCH codes, employing the coarse synchronization algorithm, as it will be explained in Section 4.1.2.1. After the cell slot timing is achieved, the frame synchronization procedure is initiated. In this second step, the secondary SCH codes must be used. Once the combination of secondary $\mathrm{SCH}$ codes used by the base station is identified, it is possible to acquire the general frame synchronization for downlink and the primary code group of cell simultaneously.

Finally, the exact primary scrambling code used by the cell is determined in the third step. This search is limited to the set of eight different scrambling codes determined by the primary code group. The reference channel employed in this step is the P-CPICH, which is transmitted continuously over the entire cell. The P-CPICH is an unmodulated code channel, which is scrambled with the cell-specific primary scrambling code of the cell. The P-CPICH is unique for each cell. After the primary synchronization code has been identified, the cell search procedure is finished and it is possible to apply the general fine synchronization algorithm in downlink with the P-CPICH channel. At the same time, the P-CCPCH is demodulated in order to extract the specific parameters necessary for user's demodulation, which are the channelization code, spreading factor, and the specific timing delay, for the downlink, and the scrambling and channelization codes, spreading factor, and DPCCH format, for the uplink. The combination of the cell search procedure and extraction of user's specific information is denoted as set-up stage of the modem.

Unlike downlink, each user has a specific synchronization reference in the uplink. If the modem knows the parameters of active users for uplink (obtained in the downlink set-up stage), the synchronization scheme is very simple. For each user, the timing reference is extracted from the DPCCH, applying the coarse and fine synchronization algorithms directly.

\subsubsection{Synchronization algorithms}

The timing information of the transmitted frame is essential in order to properly demodulate the despread signal. Even if there is a single chip duration error, the received spread spectrum signal cannot be properly demodulated.

Once the used codes in physical channels have been obtained, the appropriate timing reference is extracted. This synchronization issue is resolved following a two-step approach [20]. Firstly, coarse synchronization or initial code acquisition accomplishes the synchronization of the received signal and the corresponding code, with an uncertainty of half a chip period $\left( \pm T_{c} / 2\right)$. Secondly, fine synchronization or code tracking performs and maintains the synchronization between the received signal and the code with a precision always lower than half a chip period.

To perform the synchronization, the scrambling code properties are used. These codes have an autocorrelation function that reaches its maximum when the code and the received signal are aligned.

\subsubsection{Coarse synchronization}

As stated before, the objective of the coarse code synchronization is to achieve an initial code acquisition between the received signal and the corresponding scrambling code. This is equivalent to matching the phase of the spreading signal with the code.

There are different general acquisition techniques $[19,20$, 21]. In the serial search, all the possible phases are tested one by one sequentially. The complexity for this method is quite low but the associated acquisition time is high. In the parallel search, all the possible phases are tested simultaneously. The complexity is higher but the acquisition time is much lower than in the serial search. An intermediate approach between the serial and parallel search strategies has been implemented in order to achieve the coarse synchronization with a moderate computational load, considering the complexity versus acquisition trade-off. A study of the computational load required by the different implementation approaches is shown in Table 3.

Considering the capacity of the used DSP's, the threebranches serial-parallel approach has been implemented. The block diagram of the coarse synchronization stage is shown in Figure 9.

In the figure, several blocks can be distinguished: correlators, thresholds generator, signal control modules, and a scrambling code generator. The received match-filtered signal is correlated with different cycle-delayed code versions. The maximum correlation value from the branches is compared with the first threshold $\gamma_{1}$ which is obtained taking into account the second maximum correlation value. In order to 


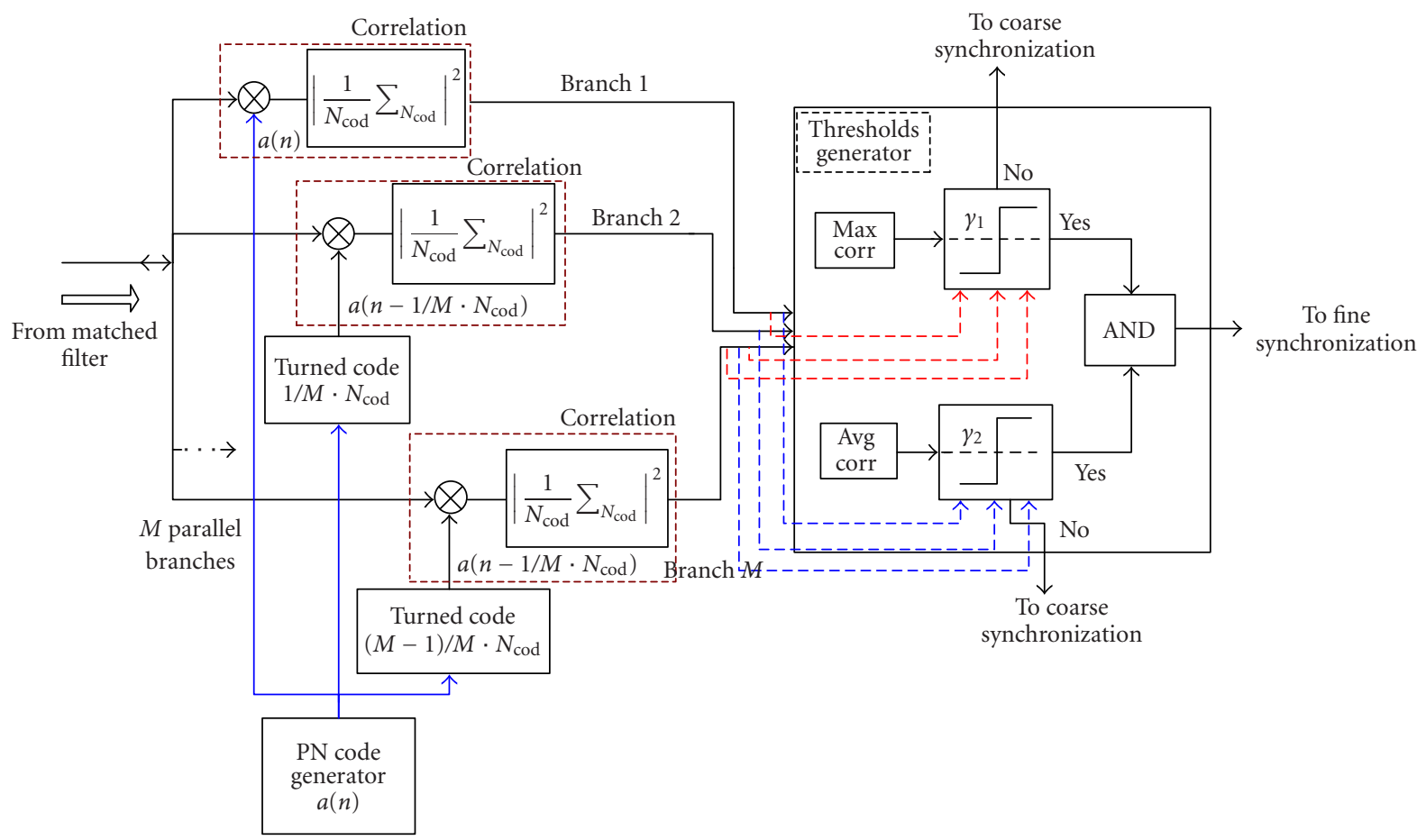

FIGURE 9: Block diagram of coarse synchronization.

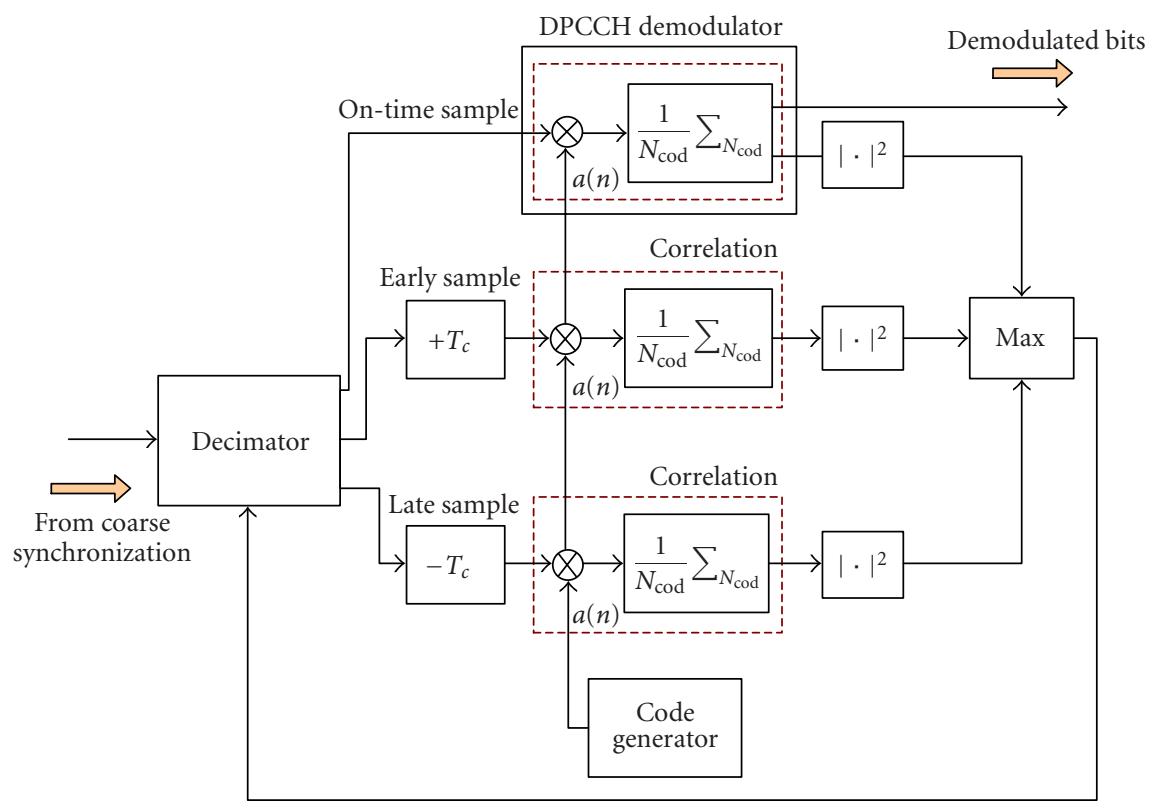

FIGURE 10: Block diagram of fine synchronization.

avoid situations in which the background noise may cause a wrong correlation which exceeds the first threshold, it is necessary to set another threshold to minimize this effect. This second threshold $\gamma_{2}$ is calculated from the average of all the correlations except the maximum value. If the input signal surpasses both thresholds, then it is coarse-synchronized and fine synchronization is triggered.

\subsubsection{Fine synchronization}

The purpose of code tracking is to perform and maintain the synchronization. Code tracking starts its operation only after coarse synchronization has been achieved. After coarse synchronization, a small phase error is still present. In order to correct this error, the loop structure shown in Figure 10 is used [19]. 


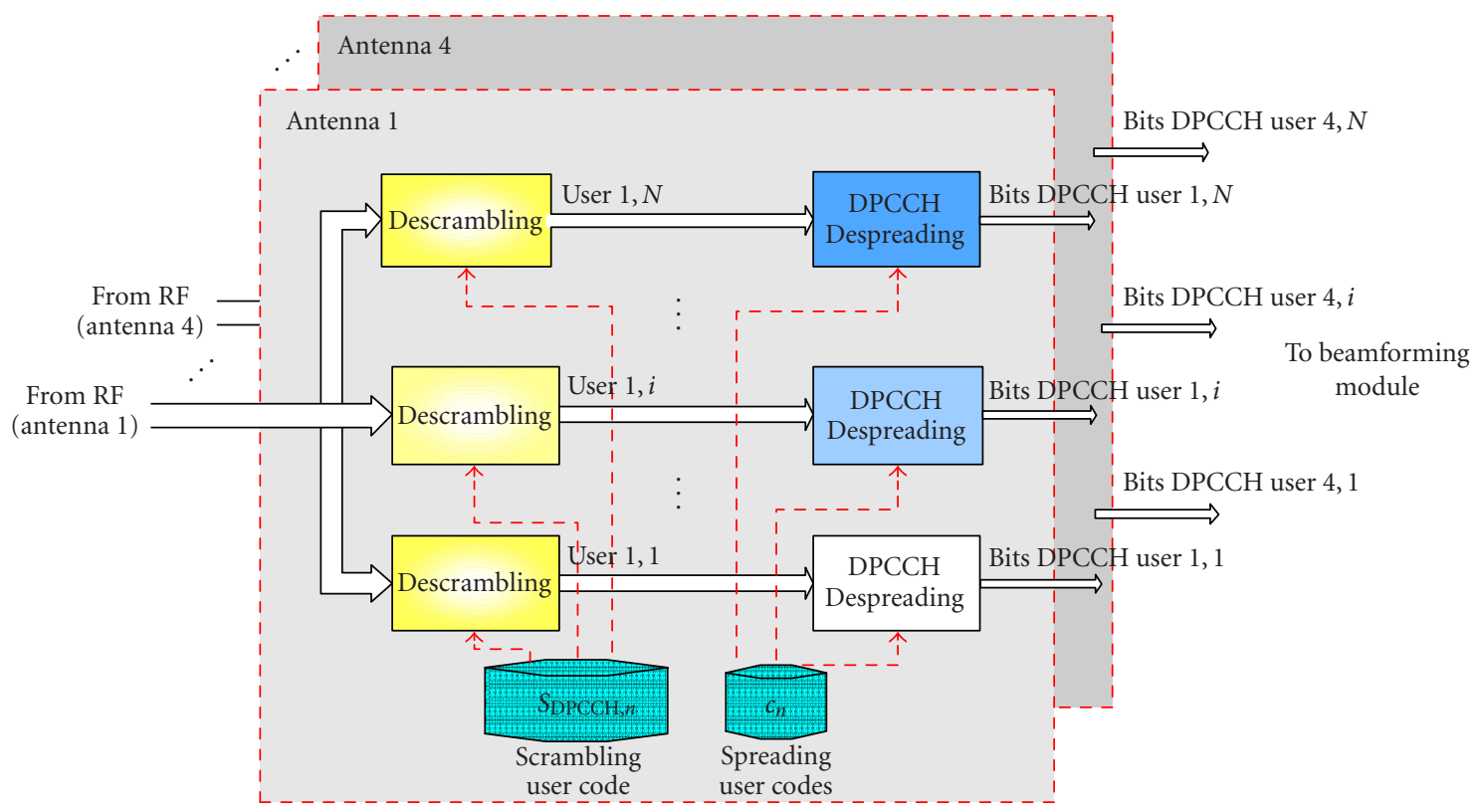

FIGURE 11: Uplink demodulator diagram.

The first block is a decimator that selects the correct sample at the right time, depending on the correlation value. In the second step, the decimated signal is delayed or advanced half a chip period, creating the late, early, and on-time branches. These three signals are correlated with the locally generated scrambling code, and the maximum absolute value of the correlations is selected. According to this selection, the timing information is updated.

\subsubsection{Demodulation in uplink and downlink}

Once the timing information and scrambling and channelization codes are determined, any UMTS physical channel can be demodulated.

In the uplink, DPCCH is demodulated for each user in order to extract the pilot bits that will be used as the reference signal in the beamforming process. To complete this task, two operations must be carried out: the complex-valued signal is descrambled by a complex-valued scrambling code $S_{\mathrm{DPCCH}, n}$ which identifies a user, and the signal is despread using the channelization code $c_{n}$ which identifies the DPCCH channel. This process is shown in Figure 11.

In the downlink, the dedicated physical channel (DPCH) is demodulated. Firstly, the signal from Node B is descrambled by a complex-value scrambling code $S_{d l, n}$ which identifies the cell and afterwards, the signal is despread through the correlation with a real-valued channelization code $c_{c h, S F, n}$ which identifies the user in the downlink. Both time-multiplexed DPCCH and DPDCH (dedicated physical data channel) bits are obtained after this operation. Once the DPCH bits for every user have been demodulated and beamformed, the spreading operation is performed with $c_{c h, S F, n}$ and scrambled with $S_{d l, n}$. The block diagrams of the modem for the downlink are shown in Figures 12a and 12b.

\subsection{Adaptive beamformer}

Immediately after the synchronization has been achieved, the following stage is the adaptive beamforming. The aim of this module is to calculate the set of array weights that make the array output signal satisfy an optimization criterion. Apart from this computation, the beamforming module adequately combines the received signal vector in order to produce a spatially filtered W-CDMA signal in the array output.

In the downlink, the base station transmits a separate beam pointing at the direction of each user, along with the broadcast channels, which are transmitted to the whole sector.

In this section, beamforming principles and implementation aspects are thoroughly explained. Moreover, theoretical expressions for the SINR are given for the operation of ADAM in uplink and downlink. In CDMA systems, this parameter is used for the estimation of capacity, throughput, and quality of service. Performance results will be shown in Section 6.1.

\subsubsection{Uplink operation and implementation}

Let $x(t)$ be the complex envelope representation for the vector of received signals in the array elements. For a situation with $K$ mobile users and one interfering source $i(t)$, the vector $\underline{x}(t)$ can be expressed as follows:

$$
\begin{array}{r}
\underline{x}(t)=\sum_{k=1}^{K} \sqrt{P_{k}} \sum_{l=1}^{L_{k}} \alpha_{k l}^{U}(t) \underline{a}^{U}\left(\theta_{k l}\right) s_{k}\left(t-\tau_{k l}\right) \\
+\sqrt{P_{\text {int }}} \underline{a}^{U}\left(\theta_{\text {int }}\right) i(t)+\underline{n}(t),
\end{array}
$$

where $P_{k}$ is the power transmitted from user $k, \alpha_{k l}$ and $\tau_{k l}$ are the complex channel gains and delay of the $l$-path of the 


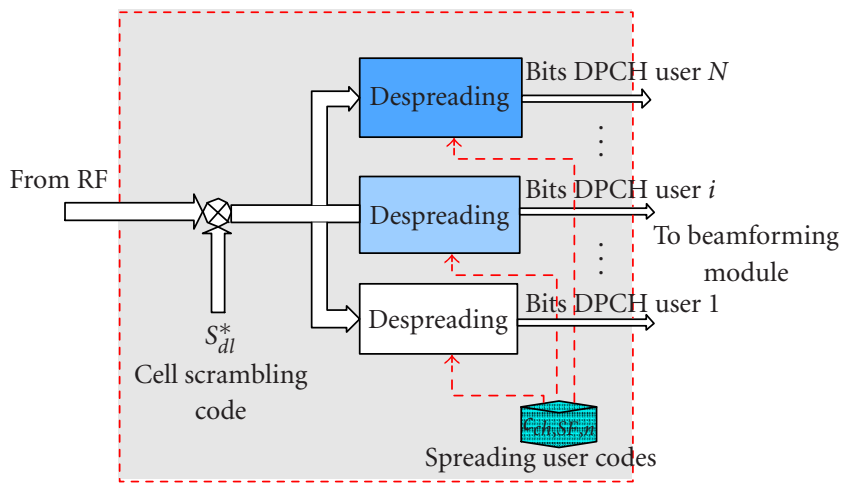

(a)

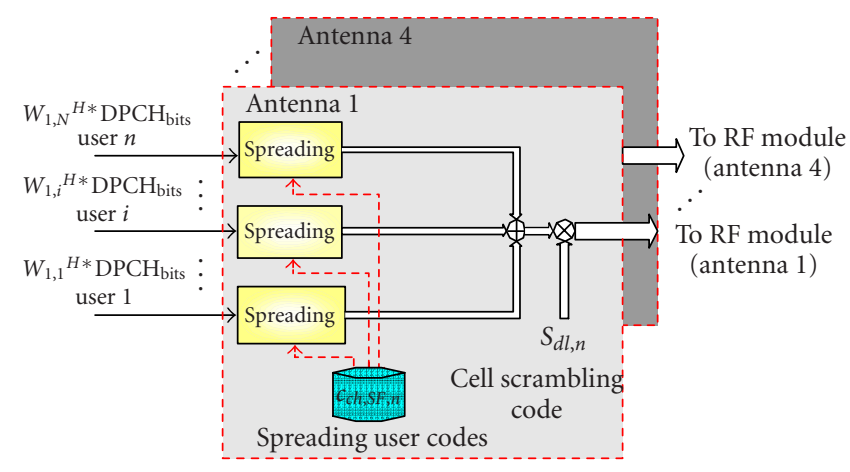

(b)

FIGURE 12: Downlink demodulator and modulator diagrams. (a) Downlink demodulator. (b) Downlink modulator.

$k$ th user, and $\underline{a}(\theta)=g(\theta) \cdot\{\exp (j(2 \pi / \lambda) d(l-1) \cos (\theta)), l=$ $1, \ldots, L\}$ is the response of a uniform linear array with $L$ antenna elements and an interelement separation $d$, such as ADAM, to a wave impinging from an azimuth direction $\theta$, including the element antenna pattern $g(\theta)$ [26]. The $k$ th user signal $s_{k}(t)$ includes modulation, data, and spreading. $P_{\text {int }}$ is the power transmitted from the external interference source. Superscript $U$ stands for the uplink. Finally, $\underline{n}(t)$ is an $L$-dimensional complex Gaussian vector with independent and identically distributed (i.i.d.) components of zero mean and variance given by the corresponding signal-to-noise ratio (SNR).

In the uplink operation, two alternatives can be considered. The first one consists in performing a total cancelation of interfering sources for each user, including the contributions from other mobile users. Let $\underline{w}_{k}$ be the uplink beamforming vector for each particular user in the total cancelation scheme. With this approach, if $K$ users are present in the cell, then a separate beamformed signal $y_{k}(t)=\underline{w}_{k}^{H} \underline{x}(t)$, $k=1, \ldots, K$, should be transferred to Node B. Therefore, $K$ separate input channels would have to interface with Node B, and ADAM operation would lose its transparent behavior.

The other alternative is to apply a common beamforming weight vector $\underline{w}$ to the composite received signal $\underline{x}(t)$ (mobile user signals plus interference sources). The approach applied to ADAM is to use a linear combination of $\underline{w}_{k}$ weights to perform the common beamforming operation that is required in the uplink. All individual beamforming vectors have a common feature, namely, the cancelation of interfering sources external to the system. Following this technique, the array output can be expressed as follows:

$$
\begin{aligned}
y(t)= & \sum_{k=1}^{K} \underline{w}_{k}^{H} \underline{x}(t)=\left\{\sum_{k=1}^{K} \underline{w}_{k}^{H}\right\} \underline{x}(t)=\underline{w}^{H} \underline{x}(t) \\
= & \underline{w}^{H}\left\{\sum_{k=1}^{K} \sqrt{P_{k}} \sum_{l=1}^{L_{k}} \alpha_{k l}^{U}(t) \underline{a}^{U}\left(\theta_{k l}\right) s_{k}\left(t-\tau_{k l}\right)\right\} \\
& +\sqrt{P_{\text {int }} \underline{w}^{H}} \underline{a}^{U}\left(\theta_{\text {int }}\right) i(t)+\underline{w}^{H} \underline{n}(t) .
\end{aligned}
$$

This scheme is called partial interference cancelation because only common interfering sources will be canceled after applying common beamforming weights. The uplink SINR in the array output for user $k$ is therefore given by

$$
\begin{aligned}
& \operatorname{SINR}_{k}^{U L} \\
& =\frac{\underline{w}^{H} E\left\{\left|\sqrt{P_{k}} \sum_{l=1}^{L_{k}} \alpha_{k l}^{U}(t) \underline{a}^{U}\left(\theta_{k l}\right) s_{k}\left(t-\tau_{k l}\right)\right|^{2}\right\} \underline{w}}{\left\{\begin{array}{l}
\underline{w}^{H} E\left\{\left|\sum_{\substack{i=1 \\
i \neq k}}^{K-1} \sqrt{P_{i}} \sum_{l=1}^{L_{i}} \alpha_{i l}^{U}(t) \underline{a}^{U}\left(\theta_{i l}\right) s_{i}\left(t-\tau_{i l}\right)\right|^{2}\right\} \underline{w} \\
+\underline{w}^{H} E\left\{\left|\sqrt{P_{\text {int }}} \underline{a}^{U}\left(\theta_{\text {int }}\right) i(t)\right|^{2}\right\} \underline{w}+\underline{w}^{H} E\left\{|\underline{n}(t)|^{2}\right\} \underline{w}
\end{array}\right\}} .
\end{aligned}
$$

The second term in the denominator represents the common interference contribution that appears in the array output. The level of common interference cancelation is given by the magnitude of $\left|\underline{w}^{H} \underline{a}^{U}\left(\theta_{\text {int }}\right)\right|^{2}$.

In both alternatives, the calculation of individual beamforming weights $\underline{w}_{k}$ fulfils the minimum mean square error (MMSE) criterion in the array output. The optimum solution is given by the Wiener-Hopf equation as $\underline{w}_{k}=R_{k}^{-1} \underline{p}_{k}$, where $R_{k}=E\left\{\underline{x}_{k}(n) \underline{x}_{k}^{H}(n)\right\}$ and $p_{k}=E\left\{\underline{x}_{k}(n) d_{k}^{*}(n)\right\}, \underline{x}_{k}(n)$ and $d_{k}(n)$ being the vector of demodulated pilot bits in the antenna array and the reference pilot bits, respectively. This equation does not represent a practical solution so that a suboptimum set of weights must be calculated by means of adaptive algorithms. This procedure is based on the iterative estimation of $\underline{w}_{k}$ each time a new pilot bit is demodulated. In this way, the antenna is capable of adapting its radiation pattern to a fast varying environment.

Two well-known adaptive algorithms have been considered, namely, NLMS and RLS, whose update equations are shown in Figures 13a and 13b, respectively. The first one is the NLMS, which is based on the instantaneous estimation of $R_{k}$ and $\underline{p}_{k}$, and only vector operations must be performed. Due to its simplicity and reduced computational complexity of $\mathrm{O}(\mathrm{L})$, NLMS is very suitable to a practical implementation that must comply with real-time requirements. 


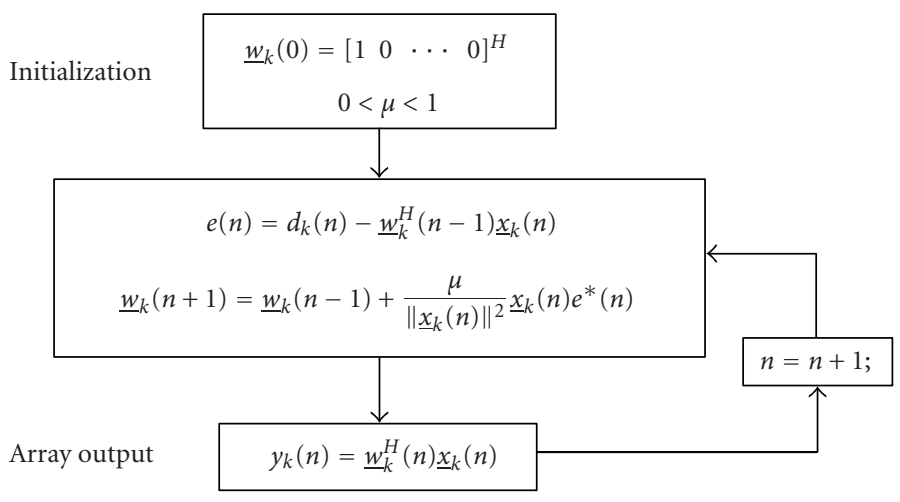

(a)

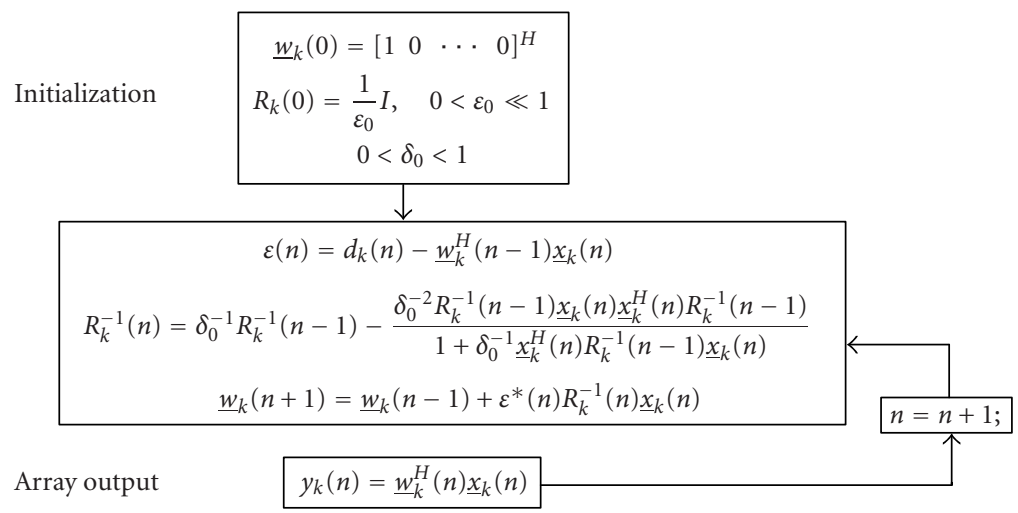

(b)

FIGURE 13: Update weight equations. (a) NLMS algorithm. (b) RLS algorithm.

On the other hand, RLS is based on the iterative estimation of the autocorrelation matrix $R_{k}$, which imposes a higher computational load than NLMS, although its convergence speed is faster. Matrix operations make RLS unfeasible for real-time implementations, being the complexity of $\mathrm{O}\left(\mathrm{L}^{2}\right)$. Further information on these algorithms can be found in $[17,18]$.

Regarding the implementation aspects, Figure 14 shows the beamforming structure used in the uplink. Demodulated DPCCH bits from the modem and the received signal vector $\underline{x}(t)$ are the inputs of the beamforming module. These bits are used to acquire the slot synchronization, which is necessary to obtain the correct pilot bits that have to be used as the reference signal in the weight computation process. After that, the single-user weights are computed, and the common beamforming weight vector is calculated. The output of the beamforming process is obtained by multiplying the received signal vector by the common weight vector and combining the resulting signal vector, without the need of demodulating each user's data channels. It must be noticed that the signal vector impinging in the array antenna is composed of contributions from several users so that both the slot synchronization and the single-user weight computation must be executed in parallel for every serviced user.

\subsubsection{Downlink operation and implementation}

Optimal downlink beamforming will minimize the interference received by other users and will enhance the useful signal power received by the desired user. Due to the frequency translation that appears in a frequency division duplex (FDD) system, uplink and downlink communication scenarios are different. As a consequence of the lack of downlink channel information, the calculation of transmission weights is all but an easy task.

One of the methods for estimating downlink weights from uplink channel information is the use of the uplink spatial covariance matrix [27]. However, this approach involves the use of many computational resources.

In order to reduce the complexity of the implementation, ADAM uses individual uplink weights as transmission vectors. Due to frequency translation $(\Delta f=190 \mathrm{MHz})$, the transmitting array factor is similar but not the same as in the uplink. Therefore, the synthesized beam will not be pointing exactly in the direction of the mobile user, and the interference received by other user will be reduced but not minimized.

For downlink, after joint transmission of the weighted signals bounded for the $K$ users from the base station, the 


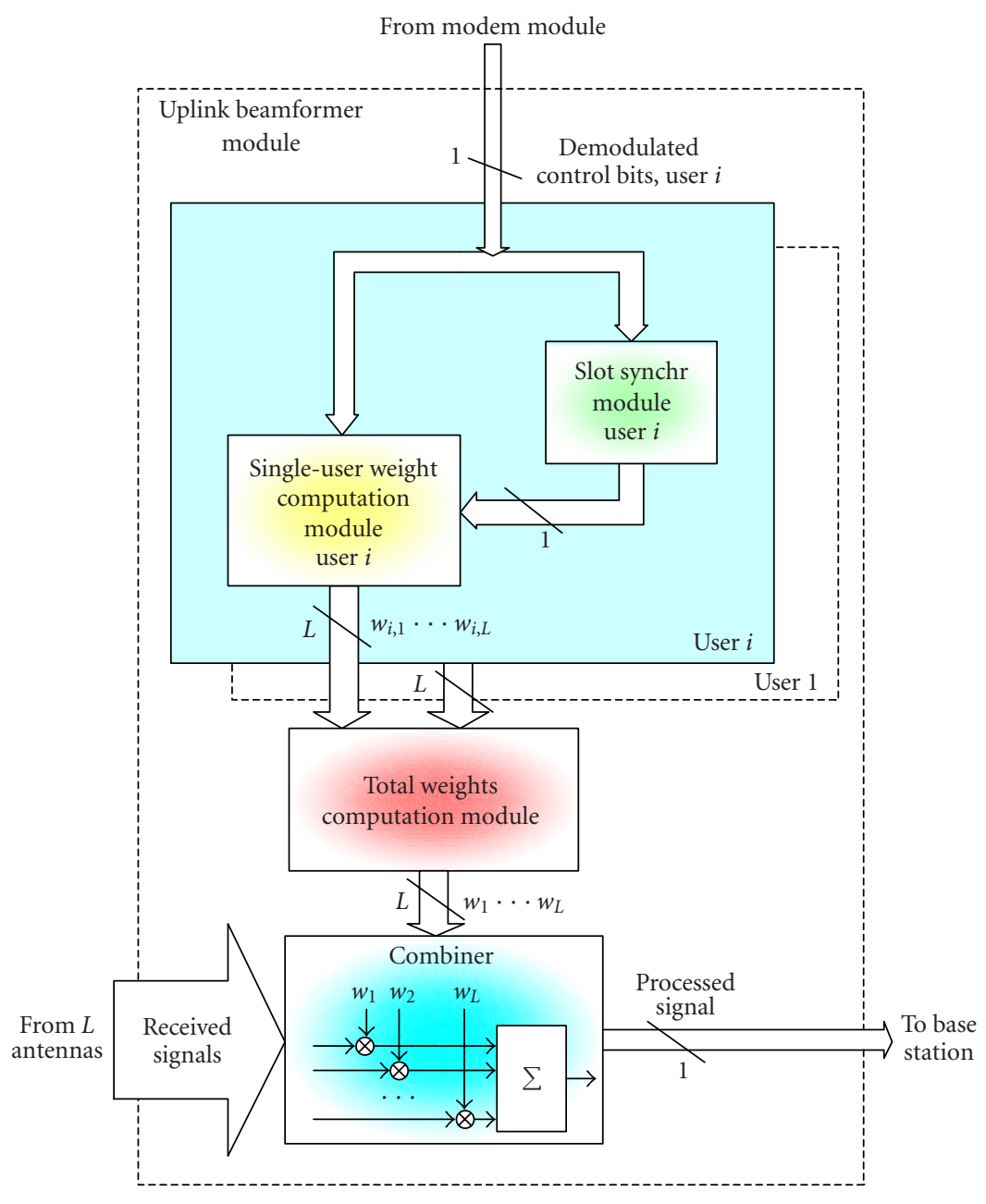

FiguRE 14: Beamformer structure: uplink.

baseband signal received by the mobile $k, x_{k}(t)$, is given by

$$
x_{k}(t)=\sum_{k=1}^{K} \sqrt{P_{k}} \underline{w}_{k}^{H} \sum_{l=1}^{L_{k}} \alpha_{k l}^{D}(t) \underline{a}^{D}\left(\theta_{k l}\right) s_{k}\left(t-\tau_{k l}\right)+n_{k}(t),
$$

where $\underline{w}_{k}$ is the transmission beamforming vector for user $k$, $P_{k}$ is the power assigned to the user $k$ signal, and $n_{k}(t)$ is a complex white Gaussian process that represents the thermal noise contribution in the mobile user equipment. The other elements of (4) have the same meaning as in (1). In an FDD system, uplink $\left(\alpha_{k l}^{U}\right)$ and downlink $\left(\alpha_{k l}^{D}\right)$ fading coefficients are uncorrelated, and in the simulations, they have been generated from independent Rayleigh fading processes.

The SINR perceived by the user $k$ can be expressed as follows:

$$
\begin{aligned}
& \operatorname{SINR}_{k}^{D L} \\
& =\frac{\underline{w}_{k}^{H} E\left\{\left|\sqrt{P_{k}} \sum_{l=1}^{L_{k}} \alpha_{k l}^{D}(t) \underline{a}^{D}\left(\theta_{k l}\right) s_{k}\left(t-\tau_{k l}\right)\right|^{2}\right\} \underline{w}_{k}}{E\left\{\left|\sum_{\substack{i=1 \\
i \neq k}}^{K-1} \underline{w}_{i}^{H} \sqrt{P_{i}} \sum_{l=1}^{L_{k}} \alpha_{k l}^{D}(t) \underline{a}^{D}\left(\theta_{k l}\right) s_{i}\left(t-\tau_{k l}\right)+n_{k}(t)\right|^{2}\right\}} .
\end{aligned}
$$

In contrast to the uplink, a complete user separation can be performed in the downlink direction. Because of that, in the proposed downlink structure shown in Figure 15, single-user weight vectors calculated in the uplink are applied as transmit beamforming weights to each user separately. Therefore, downlink beamforming is much simpler than the uplink one since the adaptive weight calculation is not required.

However, demodulation of data and control bits for each user is required, and downlink beamforming is applied at the bit level. This fact results in a considerable reduction in the computational load, as far as the multiplier submodule is concerned, in comparison to the equivalent module for the uplink. Moreover, a total cancelation of interferences is achieved thanks to the individual user separation. Nonetheless, this scheme increases the complexity of the modulation and demodulation module, as it must be performed for every user in every element of the antenna array.

In contrast to dedicated channels, broadcast information conveyed by common transport channels must be received by all the users in the cell. Therefore, the associated 


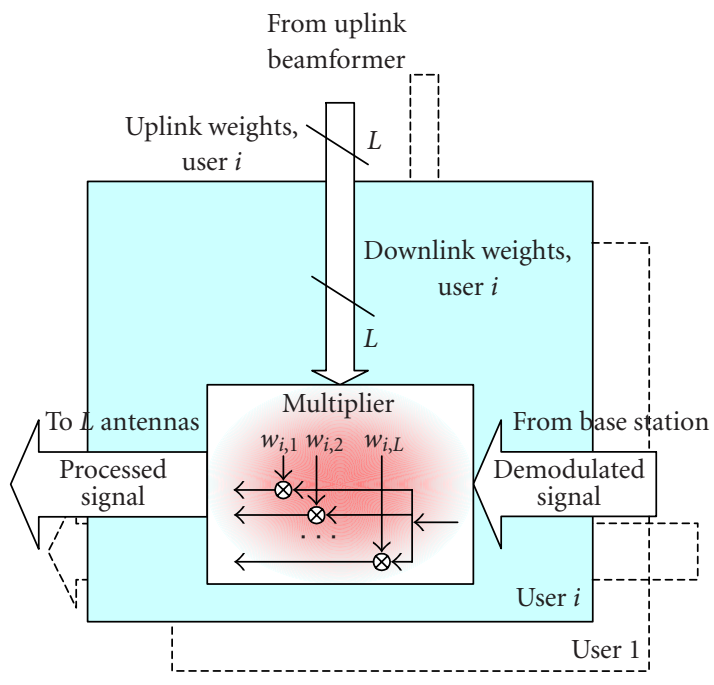

FIGURE 15: Beamformer structure: downlink.

physical channels are transmitted to the whole sector through one of the antenna array elements, which is equivalent to the radiation pattern of a conventional sectored antenna.

\section{CODE OPTIMIZATION AND LOAD DISTRIBUTION}

\subsection{Maximum number of instruction per DSP}

The analog received signal is sampled in the ADC to four samples per chip rate, obtaining packets of 1024 samples. Therefore, the number of samples per DPCCH bit can be calculated as follows:

$$
\begin{aligned}
& \text { Number of samples per DPCCH bit } \\
& \qquad=1 \mathrm{bit} \cdot 256 \mathrm{chips} / \mathrm{bit} \cdot 4 \text { samples/bit } \\
& =1024 \text { samples/bit. }
\end{aligned}
$$

For real-time execution, the allowable time for processing each packet of 1024 samples is one DPCCH bit period, that is, 66.67 microseconds.

Each of the used DSP has a performance capability of up to 2400 MIPS on pipeline. Their architecture has eight highly independent functional units (six ALUs(arithmetic and logical units) of 32-/40-bits and two 16-bit multipliers). Therefore, eight 32-bit instructions per cycle can be executed. The clock rate is $300 \mathrm{MHz}$. As a result, the number of DSP cycles for processing one bit is

$\operatorname{Num}(\text { cycles } / \text { bit })_{\text {MAX }}=$ MIPS $\cdot T_{b}=300 \cdot 66.67 \mu \mathrm{s}=20000$.

Therefore, the number of clock cycles in all the modules should be lower than 20000 cycles per bit. Table 4 shows the number of clock cycles per bit of each module. These values are always lower than the maximum number of clock cycles per bit.

\subsection{Code optimization and module load}

For code optimization, the following steps have been followed [28]:

(1) C-code writing;

(2) obtaining a maximum instruction reduction;

(3) use of DSP intrinsic operations.

The flow diagram in Figure 16 summarizes the previous steps.

These intrinsic operations belong to a special C62x DSPLIB library. It consists of some optimized functions for fixed-point DSPs. These functions are especially used in realtime applications since their execution time is much lower than the $\mathrm{C}$ equivalent code. The main disadvantage is that they can only be used under certain restrictive conditions.

After code optimization and programming, module load has been measured. Table 5 shows the complexity of each optimization step in clock cycles, time, and clock cycles per second for the module of coarse synchronization. As it can be seen, the complexity of coarse synchronization module has been reduced two orders of magnitude after the third optimization step.

Table 4 illustrates the computational load of the other modules when the three optimization steps have been applied. The reduction in clock cycles of the other modules is, on average, two orders of magnitude too.

\subsection{Load distribution in processors}

According to the required computational capacity for each module after code optimization, the distribution of load and tasks between DSPs must be carried out.

As presented in the Section 3, six Quads boards have been used for signal processing, with four DSPs each. In order to properly design the load distribution between DSPs, several questions have been considered. To begin with, it has been taken into account that two independent polarizations should be processed, so the number of available DSPs for each one is 12. Despite this independence, the load cannot be divided into three Quads per polarization. This is due to the need of five broadband receiver channels plus ADC and five broadband transmitter channels plus DAC per polarization. Receivers and transmitters boards consist of two channels each, which have to be associated to two DSPs in the same Quad. As a result, receiving and transmitting channels must be considered as pairs so that the possibility of using three independent Quads per polarization is eliminated.

Another point to take into consideration is the association of a task per DSP as far as possible. In this way, not only the load distribution but also the data exchange between DSPs is more easily understood. As regards the data exchange between DSPs and Quads, the tasks and load distribution has been designed aiming at reducing the number of data transfers between processors as much as possible. This makes the interconnection between DSPs simpler since less synchronization for data exchange is needed. Data transfers between DSPs are preferred to those between Quads due to the higher complexity of transfer and synchronization in the last ones. 
TABLE 4: Computational load of synchronization, demodulation, and beamforming modules.

\begin{tabular}{l|cccc}
\hline Module & Clock cycles/bit & Time $(\mu$ s) & $\begin{array}{c}\text { Million of clock cycles } \\
\text { per second }\end{array}$ & $\begin{array}{c}\text { DSP capacity } \\
\text { used }(\%)\end{array}$ \\
\hline Coarse synchronization & 8700 & 29 & 131 & $43.5 \%$ \\
Fine synchronization and demodulation & 6600 & 22 & 99 & $33 \%$ \\
Slot synchronization & 890 & 3 & 13 & $4.43 \%$ \\
Single-user weight computation & 830 & 3 & 13 & $4.17 \%$ \\
Common weight computation & 5200 & 17 & 78 & $26 \%$ \\
Combiner & 11500 & 38 & 174 & $58 \%$ \\
\hline
\end{tabular}

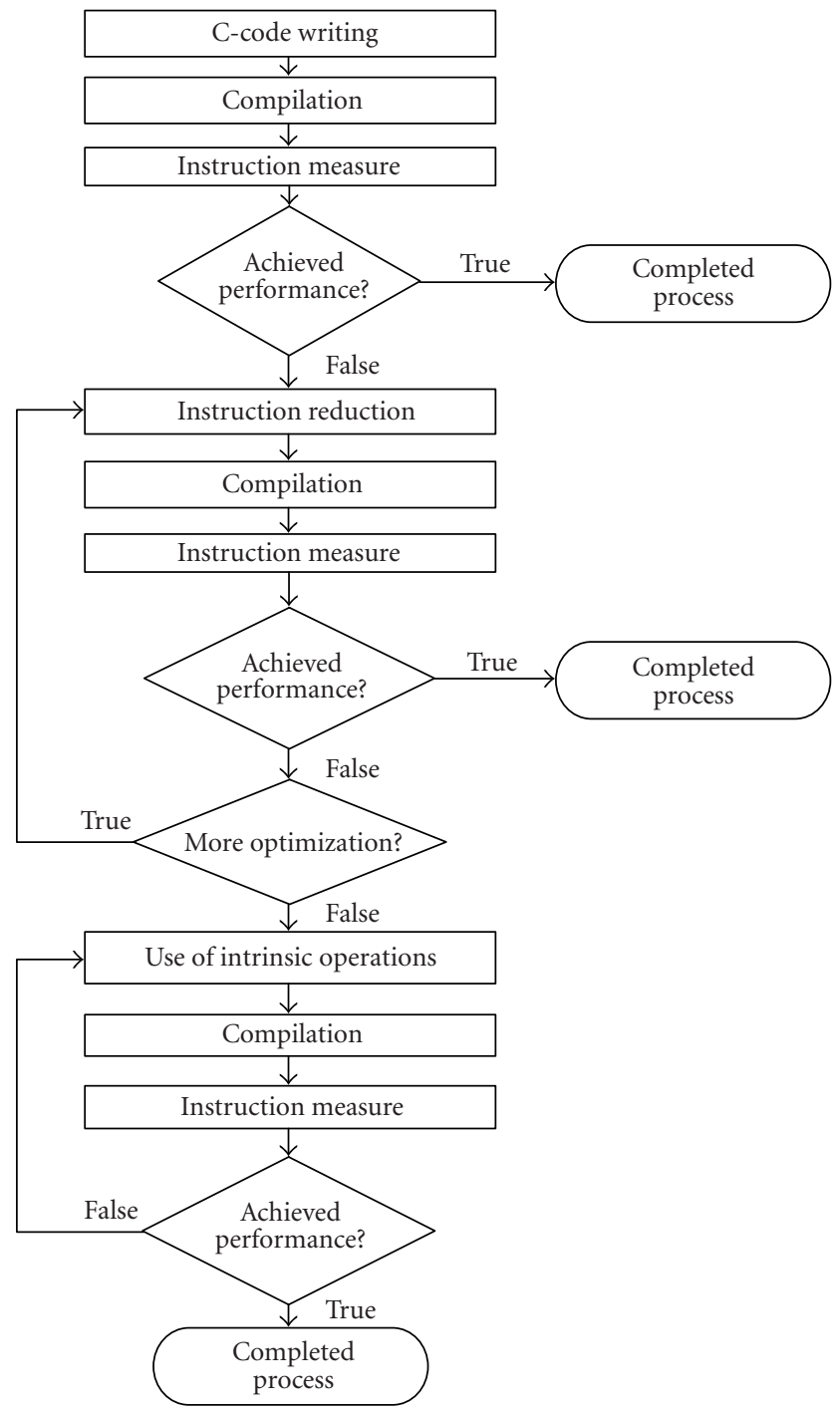

Figure 16: Optimization steps flow diagram.

On this basis, the scheme in Figure 17 is proposed. As it can be observed, the synchronization and demodulation of received signals from the four antennas in the uplink are processed in the same Quad, hence avoiding the extra data exchange and the difficulties of making a correct synchronization if the signals would be received in different Quads. Similarly, all the transmitted data are obtained and sent to the antennas in a single Quad, for the downlink. Due to computational cost restrictions, only three users can be processed with this hardware implementation. A higher number of users could be processed with more DPSs or with higher computational capacity ones.

\section{RESULTS}

\subsection{Simulations results}

Simulations have been conducted to obtain uplink and downlink performance results. Several aspects and characteristics have been varied in order to study different possible implementations.

Concerning adaptive algorithms, the performances achieved with two of them have been studied. These algorithms are NLMS and RLS, which have been widely used in adaptive array processing applications [17]. However, due to computational load restrictions, only NLMS has been implemented in the first version of the prototype.

As it was said in the introduction, the operation of ADAM must be completely transparent to Node B. This approach has an impact on the performance achieved with the adaptive beamformer. In the first group of simulations, a single-cell scenario with a variable number of mobile users is studied, including the effect of external interference on system performance. Afterwards, system-level simulation results show the capacity increase obtained with ADAM, compared to a conventional sector antenna.

\subsubsection{Uplink simulation results}

As explained in Section 4.2, two cancelation schemes have been considered in the uplink: total interference cancelation and partial interference cancelation. Performance obtained with both schemes has been studied by means of simulation.

Figure 18 shows the performance achieved by both cancelation schemes when an external interference source is present.

As it can be observed, both array factors cancel the external interference contribution. However, if the total cancelation scheme is used, contributions from other mobile users 
TABLE 5: Reduction of the number of clock cycles in the coarse synchronization module.

\begin{tabular}{c|cccr}
\hline Module & Optimization step & Clock cycles/bit & Time $(\mu$ s $)$ & Millions of clock cycles per second \\
\hline \multirow{3}{*}{ Coarse synchronization } & 1 & 2571296 & 8571 & 38500 \\
& 2 & 65732 & 219 & 986 \\
& $\mathbf{3}$ & $\mathbf{8 7 0 0}$ & $\mathbf{2 9}$ & $\mathbf{1 3 1}$ \\
\hline
\end{tabular}

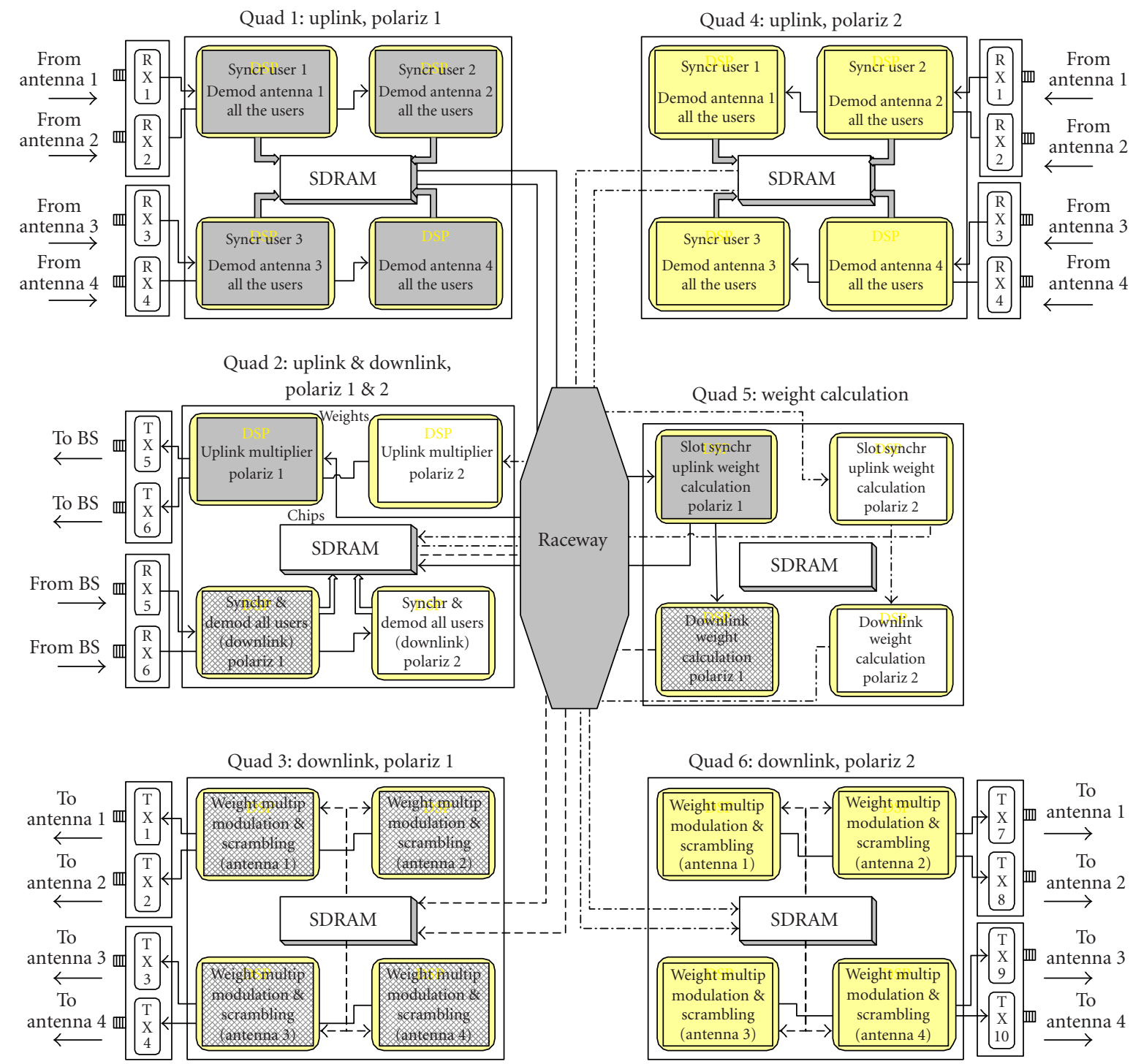

Figure 17: Load distribution.

will also be canceled, whereas with partial cancelation, a simultaneous pointing in the directions of mobile users appears as a result of the linear combination of $\underline{w}_{k}$. As the number of users uniformly distributed within the cell is increased, the final uplink radiation pattern tends to provide a sectored coverage.

A simulation environment with a uniform distribution of mobile pedestrian users has been studied. Mobile speed is
$3 \mathrm{~km} / \mathrm{h}$, and multipath fading is given by the two-path profile proposed in [2]. In (1), $\theta_{k l}$ is characterized by a Laplacian azimuth spectrum along with a Gaussian distribution for each user, with an angular spread of $10^{\circ}$. The number of rays impinging on the array per user is found as a Poisson random variable with a mean value of 25 [29]. Each user transmits only one data channel, with a spreading factor of 64 . 


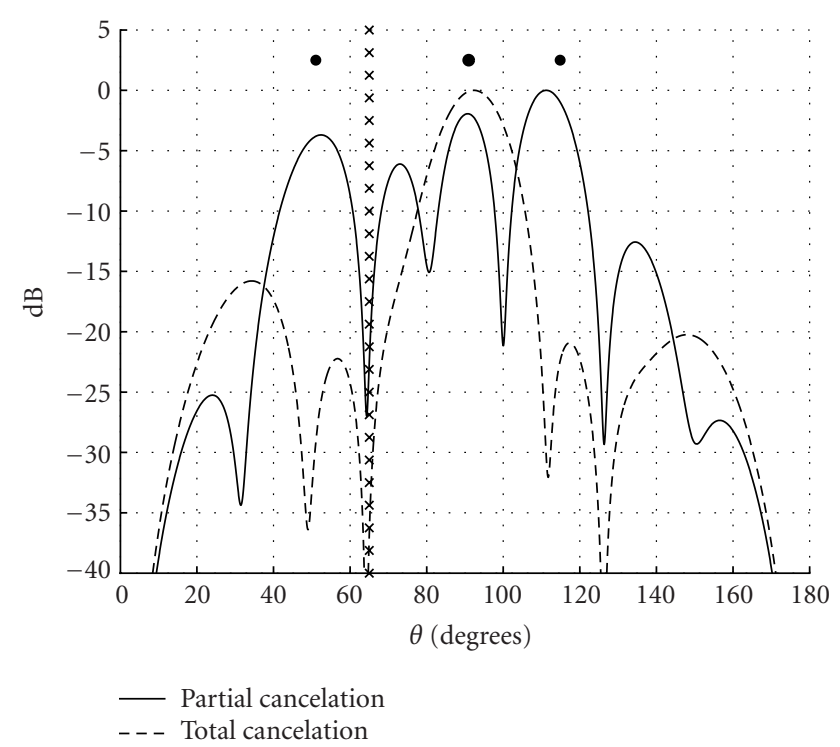

FIGURE 18: Normalized array factor for total and partial cancelation schemes $(\bullet$ : mobile users, $\times$ : external interference).

In the simulations, a perfect power control algorithm is assumed for mobile users, that is, $P_{k}=P$, and external interference power $P_{\text {int }}$ is set to $F \mathrm{~dB}$ over $P$.

Figure 19 shows the average uplink SINR increase obtained with ADAM with respect to a typical sectored antenna in two scenarios: $F=-150 \mathrm{~dB}$ (only mobile users are present in the cell), and one external interference with $F=20 \mathrm{~dB}$. In the first scenario, the SINR improvement converges to $6 \mathrm{~dB}$ when the total cancelation scheme is used. With partial cancelation, ADAM will provide the same performance as the individual sectored antenna. However, in the second scenario, the partial cancelation scheme outperforms the sectored antenna in more than $5 \mathrm{~dB}$.

As it can be observed in Figure 19, RLS provides better performance than NLMS, although their behavior converges as the number of users increases. In the case of $F=20 \mathrm{~dB}$, the difference between both algorithms is mainly due to the fact that RLS provides a higher cancelation level for the external interference source.

Table 6 shows the reduction of the interference power in the array output as a function of $F$. It can be observed that as $F$ increases, both algorithms provide a more significant interference reduction. The inclusion of the strong external interference produces a spatial coloured covariance matrix because the most significant part of interfering power is concentrated around the same angular direction. As a consequence, when the number of users increases and $F$ is reduced, interference is uniformly distributed in the cell, and the level of interference cancelation is low.

\subsubsection{Downlink simulation results}

In the downlink, only the total interference cancelation scheme has been considered. Figure 20 shows the average SINR increase experimented by the mobile user when the

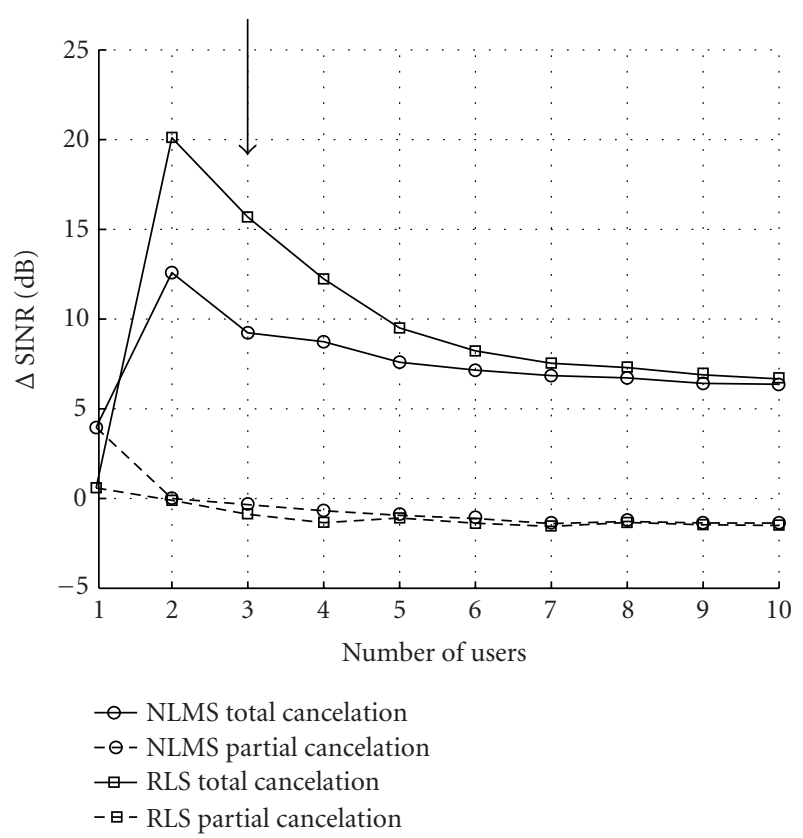

(a)

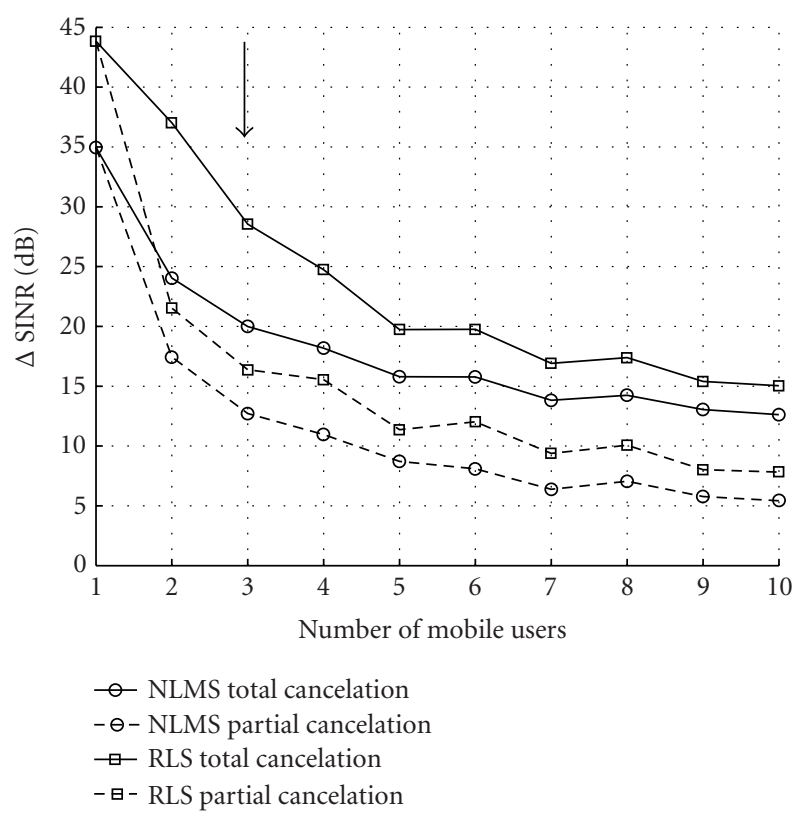

(b)

FIGURE 19: Uplink SINR increase as a function of the number of users. (a) $F=-150 \mathrm{~dB}$. (b) $F=20 \mathrm{~dB}$.

proposed downlink beamforming algorithm is used. In the downlink, NLMS provides a higher SINR increase than RLS because the improvement obtained with RLS is mainly due to the cancelation of the external interfering source, which does not influence downlink performance.

In Figures 19 and 20, the performance is studied for up to ten mobile users in the system. However, and as it was 
TABLE 6: Cancelation level of the external interference source with the partial cancelation scheme.

\begin{tabular}{c|cc|cc}
\hline \multirow{2}{*}{$F(\mathrm{~dB}) 10 \log _{10}\left(P_{\text {int }} / P\right)$} & \multicolumn{3}{|c}{ Interference cancelation level $(\mathrm{dB}) 10 \log _{10}\left(\left|\underline{w}^{H} \underline{a}^{U}\left(\theta_{\text {int }}\right)\right|^{2}\right)$} \\
\cline { 2 - 5 } & \multicolumn{3}{|c|}{$K=3$ users } & \multicolumn{2}{c}{$K=10$ users } \\
\cline { 2 - 5 } & NLMS & RLS & NLMS & -6.38 \\
\hline 150 & -5.71 & -5.52 & -5.36 & -7.83 \\
10 & -9.29 & -24.36 & -10.31 & -13.02 \\
20 & -15.47 & -37.19 & -16.00 & -24.54 \\
30 & -23.58 & -50.83 & -19.53 & -40.83 \\
\hline
\end{tabular}

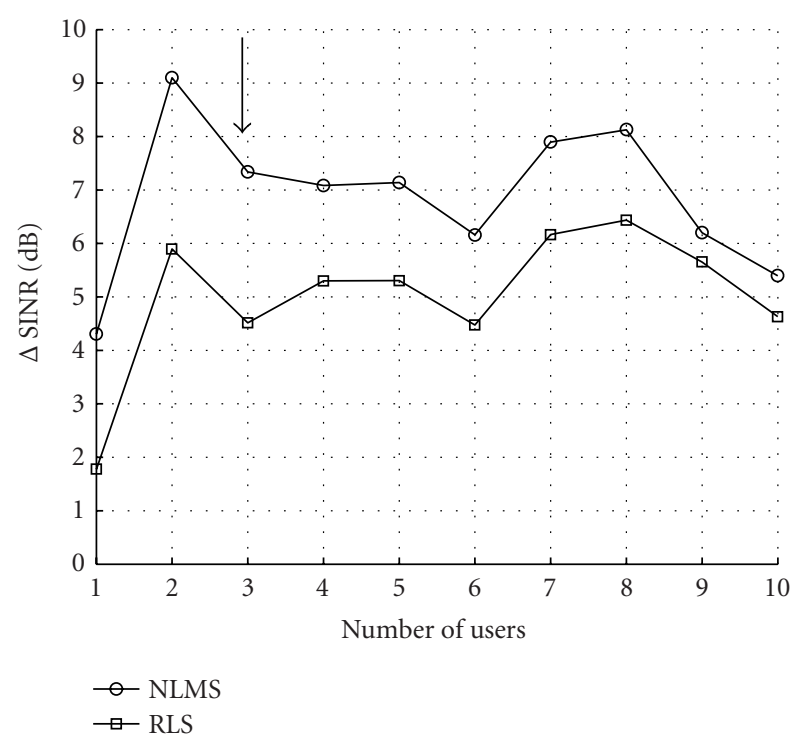

(a)

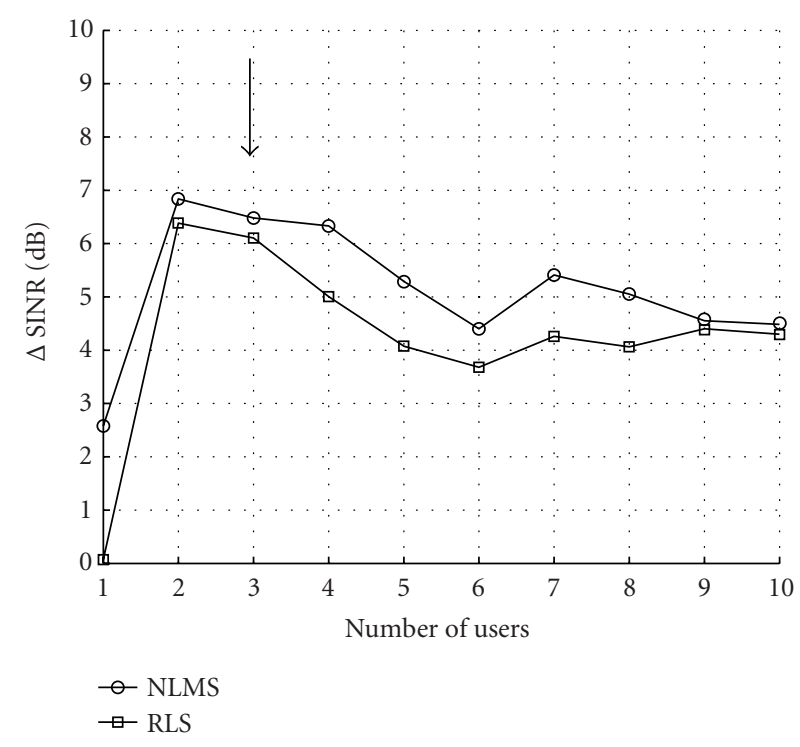

(b)

FIGURE 20: Downlink SINR increase seen by the mobile user. (a) $F=-150 \mathrm{~dB}$. (b) $F=20 \mathrm{~dB}$.

explained in Section 5.3, available processing hardware allows for processing a maximum of three mobile users. As well, due to the demanding computational load required by the RLS algorithm, the beamforming process is controlled by NLMS. From the above results, it can be concluded that, for a situation with three users and an external interfering source of $F=20 \mathrm{~dB}$, ADAM prototype provides an SINR increase of 12.5 and $6.5 \mathrm{~dB}$ over a conventional sectored antenna in the uplink and downlink, respectively.

\subsubsection{Performance in a typical user scenario}

In contrast to existing $2 \mathrm{G}$ networks, which are dominated by voice traffic, UMTS networks will provide a mixture of voice and data services with different specifications of bit rate and quality of service [30]. In addition, unlike voice, most data services are asymmetric in nature, meaning that people download more information than they send. This is typical of web browsing and streaming media services.
In order to model the mixed-service and asymmetric characteristics of UMTS networks, three different subscriber profiles are considered. The first one corresponds to a conventional voice service, with a symmetric bit rate of $12.2 \mathrm{kbps}$. The second group of subscribers deals with an asymmetric data service of $12.2 / 64 \mathrm{kbps}$, and the third group demands a 12.2/144 kbps asymmetric data service. In these conditions, the base-to-mobile link will limit the capacity of the system. However, this limitation can be overcome using ADAM prototype thanks to the total interference cancelation achieved in the downlink.

The actual capacity increase achieved using the ADAM prototype must be estimated through system-level simulations. A scenario with 19 sites and 57 sectors is considered. The distance between adjacent sites is $3000 \mathrm{~m}$. In the simulations, 2000 users have been uniformly distributed within the region of interest. Regarding the service distribution, 1000 subscribers demand a voice service, 500 users demand the low bit rate data service, and the other 500 users demand a 


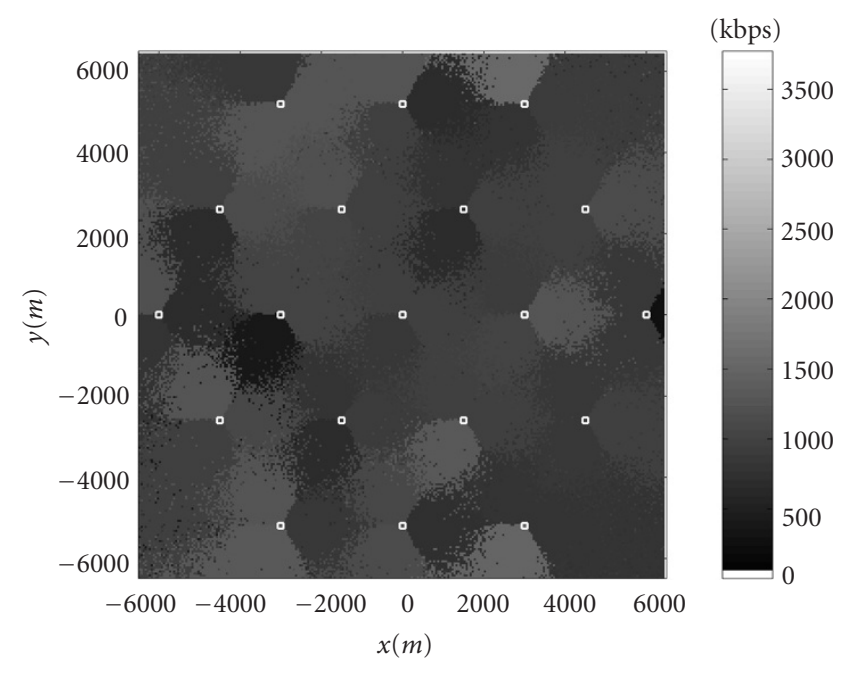

(a)

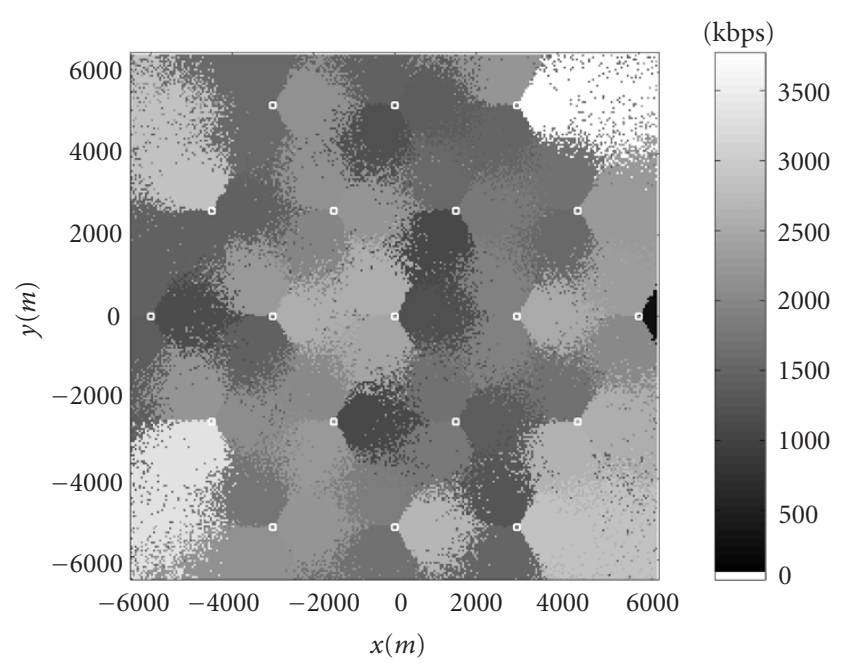

(b)

FIgURE 21: Total throughput per cell in the downlink ( $\square$ : base station site). (a) Sector antenna. (b) ADAM prototype.

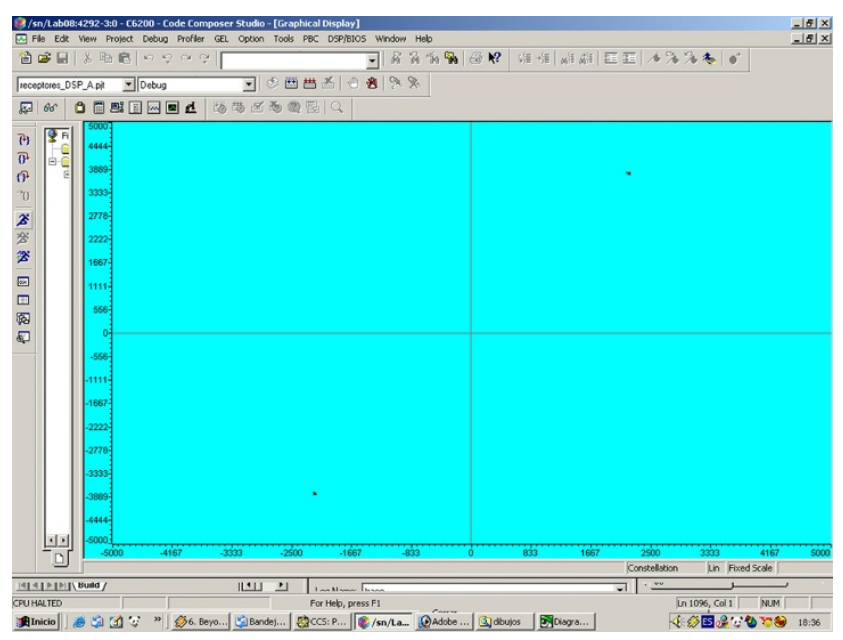

(a)

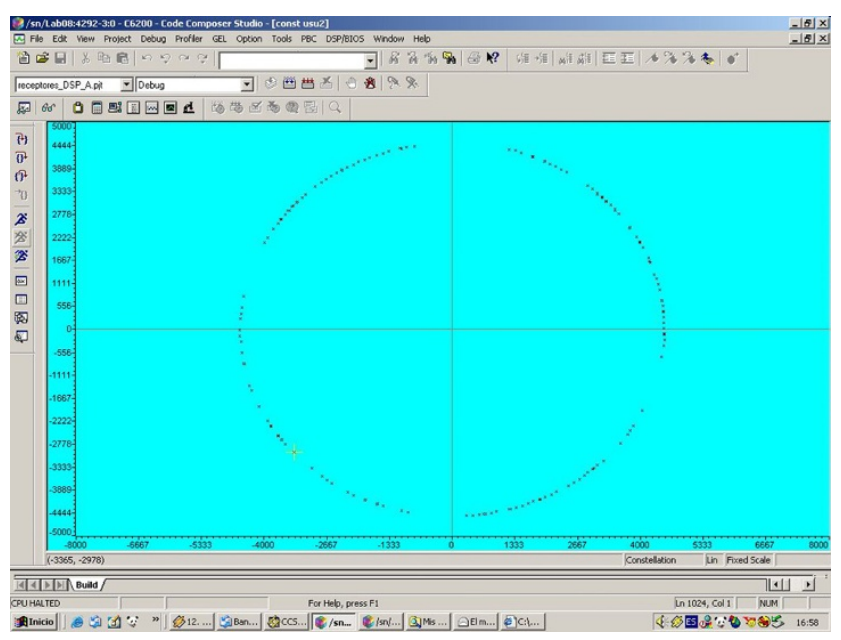

(b)

FIgURE 22: Constellation of demodulated symbols with phase and frequency offset. (a) $\omega_{0}=0, \varphi_{0}=5^{\circ}$. (b) $\omega_{0}=8.5 \mathrm{~Hz}, \varphi_{0}=5^{\circ}$.

high bit rate data service. Simulations have been performed with the network planning tool presented in [31], complemented with the incorporation of smart antennas in the scenario.

Figure 21 shows the total throughput per cell in the downlink. Using the ADAM prototype, the throughput is increased by a factor of 2 in each sector, in relation to the situation with sector antennas. This capacity increase comes from the lower number of users put to outage when the adaptive antenna is used.

\subsection{Implementation results}

One of the most important effects for the implementation is the carrier frequency error between transmitter and receiver signals. In general, the carrier error $\varphi(n)$ consists of two com- ponents [32]:

$$
\varphi(n)=\omega_{0} n T+\varphi_{0}
$$

where $\omega_{0}$ is the frequency offset, $\varphi_{0}$ is the constant phase offset, and $T$ is the symbol period.

The phase offset stays constant during the reception so that it can be compensated during the set-up stage. In contrast, the frequency offset produces the most damaging effect. Depending on its value, the total error $\varphi_{0}(n)$ varies faster. This effect is corrected modifying the received symbol phase to \pm 90 degrees since all the DPCCH symbol information is transmitted through Q channel.

The constellation of demodulated DPCCH symbols in the uplink is shown in Figure 22. Firstly, (a) illustrates a 


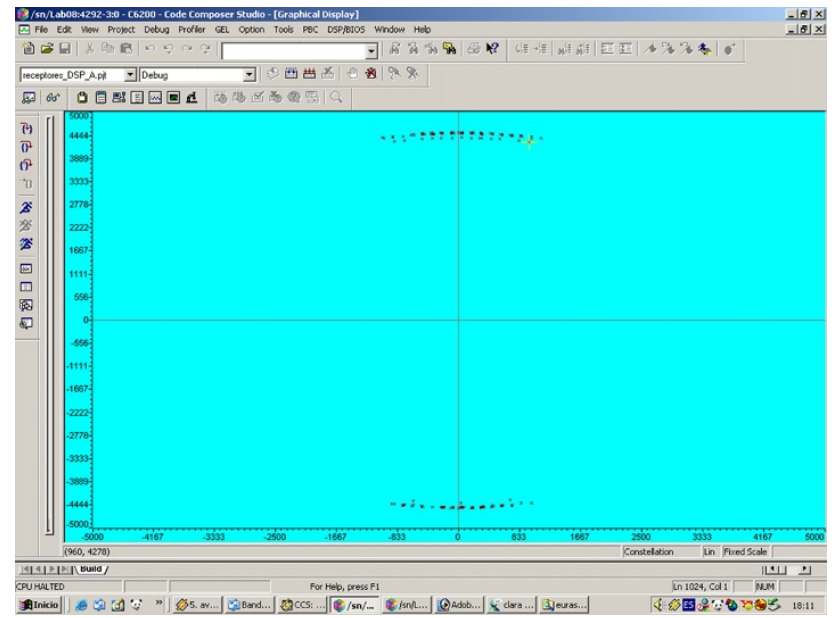

Figure 23: The constellation of demodulated symbols after phase error compensation.

situation where the phase error is 5 degrees and the frequency error is zero. In contrast, when the frequency error is not zero (Figure 22b) and no correction algorithm is applied, the phase of the received signal rotates $2 \pi$ radians each 1765 bits, that is, 117.6726 milliseconds equivalent to 11 radio frames.

Figure 23 shows the constellation after the phase correction algorithm has been applied. The phases of the symbols are not exactly 90 degrees because the implementation of the phase correction algorithm has been realized with finite resolution (sine and cosine tabulated functions). In particular, a $30^{\circ}$ resolution is considered in Figure 23 so that the maximum phase error is $30^{\circ}$. If the functions were implemented with a finer resolution, the uncertainty would be smaller. A resolution increment of the algorithm does not imply higher complexity, but it would require more memory size since more data must be stored. Depending on the memory resources, more precision could be added.

Eye diagrams are another representation for analyzing quality of demodulated symbols. Figures $24 \mathrm{a}$ and $24 \mathrm{~b}$ show the eye diagrams of noncorrected and corrected received signal, respectively. The received signal can be sampled better in the corrected case due to the improvement achieved with the phase compensation algorithm.

As an example of modulation and beamforming performance, Figure 25 shows the IF spectrum $(44 \mathrm{MHz})$ in the output of the broadband transmitters (uplink and downlink paths). As it can be seen, the spectrum corresponds to that of a W-CDMA signal, proving the transparent operation of ADAM. This signal is then upconverted to the UMTS band in the RF stages, and transferred to Node B (uplink) or to the antenna array (downlink).

Finally, the synthesized radiation pattern is plotted in Figure 26. Calculated weights are transferred in real time from the DSP blocks to the monitor-PC using LabView. This application is used in the monitoring stage to test the final radiation patterns in uplink and downlink, and to control the

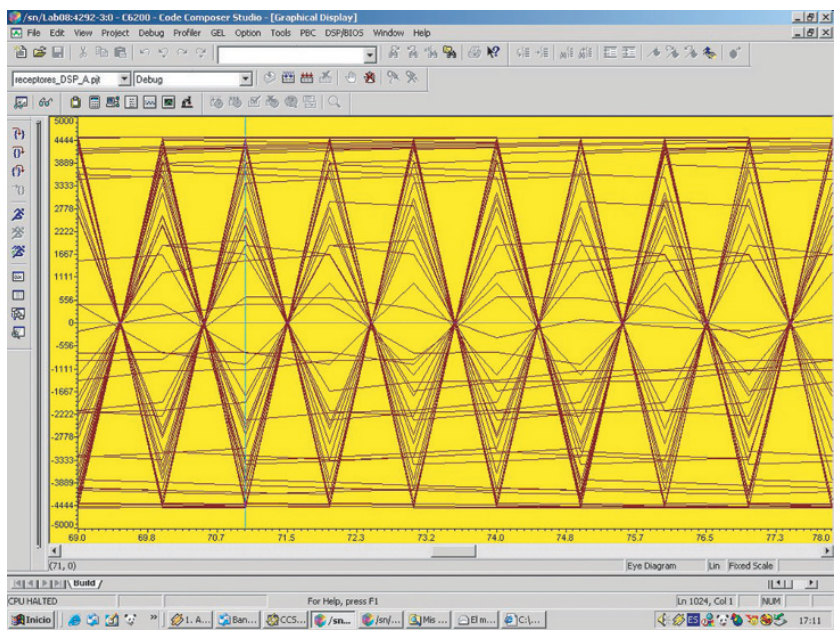

(a)

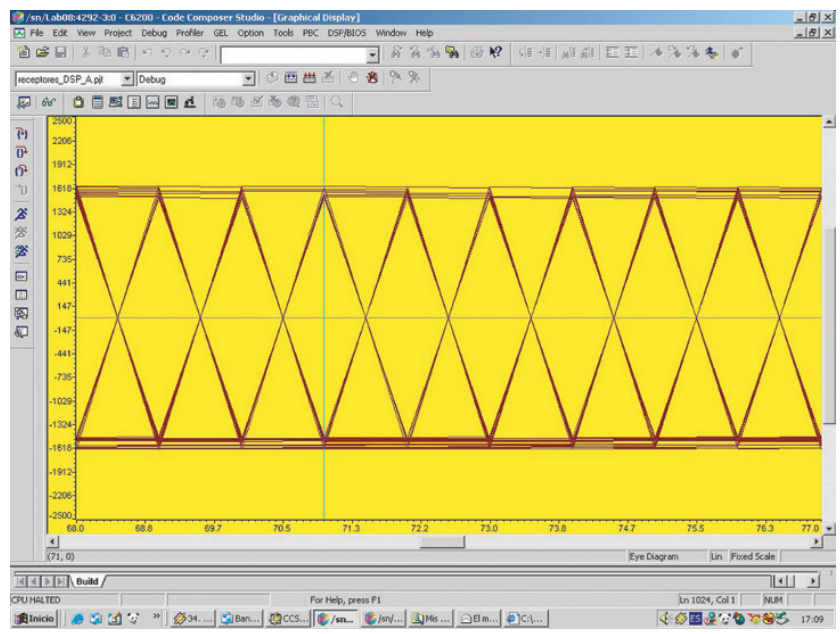

(b)

Figure 24: Eye diagrams of demodulated symbols. (a) Without phase correction. (b) With phase correction.

correct performance of the overall system, from RF parameters to SINR.

Currently, integration tests and measurements of ADAM prototype are being performed in order to characterize the behaviour of the complete system (antenna array, RF-to-IF chains, and DSP stages) in uplink and downlink.

\section{CONCLUSIONS}

A novel and real implementation of an adaptive antenna prototype for UMTS has been presented. Its main features are flexibility, modularity, and transparency in operation, which make it suitable to be connected to any existing Node B, regardless of whether it is prepared to work with an adaptive antenna or not. The focus of the contribution has been on the implementation aspects of the digital signal processing stages 


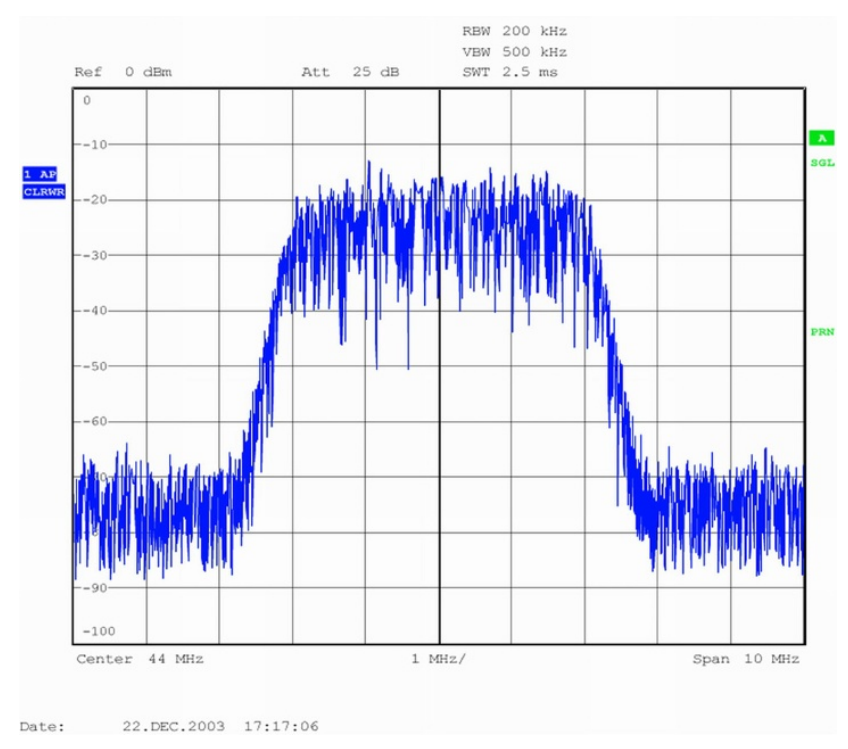

Figure 25: IF spectrum (uplink path).

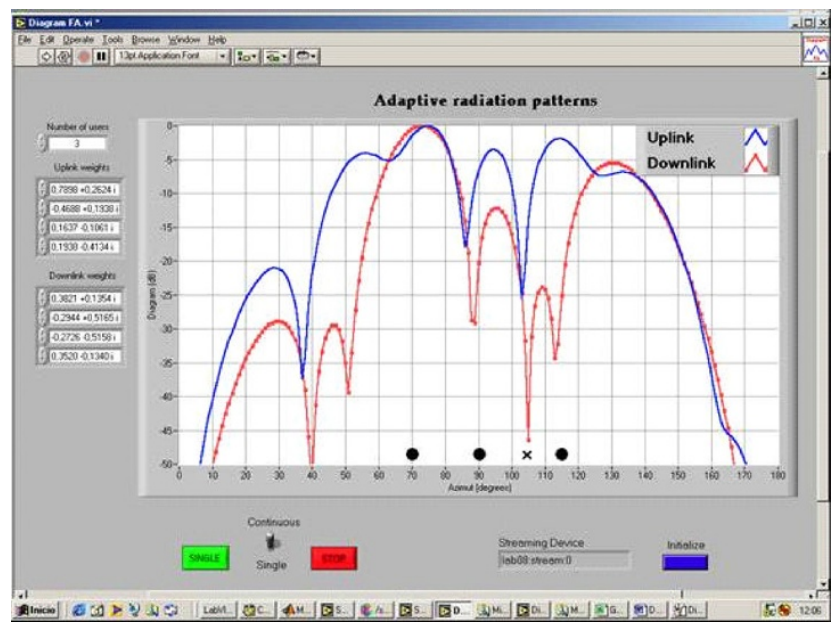

FIgURE 26: Synthesized beamformed radiation patterns $(\bullet$ : mobile users, $\times$ : external interference). Downlink diagram is shown for user in azimuth $70^{\circ}$.

(modem, synchronization, and beamforming), although an overall description of the system has been presented.

Simulation and measurement results show the feasibility and performance achieved, which outperforms the capacity obtained with a typical sectored antenna, especially in a mixed-service scenario. In the case of asymmetric traffic scenarios, ADAM prototype provides in average a $100 \%$ total throughput increase per sector in comparison with the performance of a conventional sectored antenna, as it is shown in the results of Section 6.1.3.

Further research work is currently being done on integration with RF stages. Also, the analysis and definition of measurement procedures are currently being carried out. These procedures will provide a characterization of ADAM performance in anechoic chambers and outdoor environments in connection with real cellular base stations. Moreover, a similar prototype for global system for mobile communications (GSM) standard (under the framework of Enhanced-GSM adaptiVe Antenna (EVA) project) is currently under development because our final objective is the design of a dual smart antenna for UMTS and GSM systems.

\section{ACKNOWLEDGMENTS}

The authors wish to thank Dragados Sistemas, S.A., and Vodafone for their support in the development of the ADAM project. They would also like to thank the Spanish Ministry of Science and Technology (MCYT) for the financial support of the MAIN project and all the professors and researchers involved in the ADAM project, especially, Professor Manuel Sierra-Pérez.

\section{REFERENCES}

[1] L. C. Godara, "Application of antenna arrays to mobile communications. I. Performance improvement, feasibility, and system considerations," Proceedings of the IEEE, vol. 85, no. 7, pp. 1031-1060, 1997.

[2] Third Generation Partnership Project (3GPP) Specifications, $\mathrm{ftp}: / / \mathrm{ftp} .3 \mathrm{gpp}$. org/Specs/latest.

[3] A. O. Boukalov and S.-G. Häggman, "System aspects of smart-antenna technology in cellular wireless communications-an overview," IEEE Transactions on Microwave Theory and Techniques, vol. 48, no. 6, pp. 919-929, 2000.

[4] M. Beach, C. Simmonds, P. Howard, and P. Darwood, "European smart antenna test-bed-field trial results," IEICE Transactions on Communications, vol. E84-B, no. 9, pp. 2348-2356, 2001.

[5] R. Arnott and S. Ponnekanti, "Experimental investigation of adaptive antennas in a real DCS-1800 mobile network," in Proc. 3rd ACTS Mobile Communications Summit, pp. 535-543, Rhodes, Greece, June 1998.

[6] ArrayComm Corporation, www.arraycomm.com.

[7] Kyocera Corporation, www.kyocera.com.

[8] S. Anderson, B. Hagerman, H. Dam, et al., "Adaptive antennas for GSM and TDMA systems," IEEE Personal Communications, vol. 6, no. 3, pp. 74-86, 1999.

[9] J. Mitola III, Software Radio Architecture: Object-Oriented Approaches to Wireless Systems Engineering, John Wiley \& Sons, New York, NY, USA, 2000.

[10] E. Buracchini, "The software radio concept," IEEE Communications Magazine, vol. 38, no. 9, pp. 138-143, 2000.

[11] G. Finlay, "Understanding SDR requirements," Wireless Systems Design, vol. 6, no. 7, pp. 43-50, 2001.

[12] Metawave Communications Corporation, www.metawave. com.

[13] Marconi Corporation, www.marconi.com.

[14] M. Calvo, V. Burillo, L. de Haro, and J. M. Hernando, Sistemas de Comunicaciones Móviles de $3^{\mathrm{a}}$ Generación (UMTS), Fundación Airtel Vodafone, Madrid, Spain, 2002.

[15] H. Holma and A. Toskala, WCDMA for UMTS, John Wiley \& Sons, Chichester, UK, 2nd edition, 2001.

[16] M. Sierra, M. Calvo, L. de Haro, et al., "Modular smart antenna multi-standard for multi-operator cellular communications scenarios," Patent no. P200102780, Spain, 2001. 
[17] S. Haykin, Adaptive Filter Theory, Prentice-Hall, Englewood Cliffs, NJ, USA, 3rd edition, 1996, Chapters 9 and 13.

[18] L. C. Godara, "Application of antenna arrays to mobile communications. II. Beam-forming and direction-of-arrival considerations," Proceedings of the IEEE, vol. 85, no. 8, pp. 1195 1245, 1997.

[19] H. Lim and K. Cheun, "Analysis of decimator-based fulldigital delay-locked PN code tracking loops for bandlimited direct-sequence spread-spectrum signals in AWGN," IEICE Transactions on Communications, vol. E81-B, no. 10, pp. 1903-1911, 1998.

[20] T. F. Wong, "Spreading code acquisition and tracking," in Spread spectrum and CDMA (EEL6503), University of Florida, Fla, USA, 2002, Chapter 5, http://wireless.ece.ufl. edu/eel6503/.

[21] T.-Y. Cheng and K.-C. Chen, "Joint synchronization and data detection digital receiver for direct sequence spread spectrum systems," in IEEE 47th Vehicular Technology Conference, vol. 3, pp. 2103-2107, Phoenix, Ariz, USA, May 1997.

[22] Sistemas Radiantes F. Moyano., UMTS (1900-2200 MHz) BTS Antennas, www.moyano.com.

[23] Pentek operating manuals, www.pentek.com.

[24] Mercury Computer Systems, "RACE++ Series, Eight-Port crossbar, www.mc.com.

[25] T. Ojanperä and R. Prasad, Wideband CDMA for Third Generation Mobile Communications, Artech-House Publishers, Boston, Mass, USA, 1998.

[26] C. A. Balanis, Antenna Theory: Analysis and Design, John Wiley \& Sons, New York, NY, USA, 2nd edition, 1997.

[27] H. Dai and L. Mailaender, "Performance comparison of CDMA downlink transmission techniques with power control," Technical memorandum, Bell Labs, Lucent Technologies, 2000.

[28] Texas Instruments, TMS320C6000 Optimizing Compiler User's Guide, www.ti.com.

[29] K. I. Pedersen, P. E. Mogensen, and B. H. Fleury, "A stochastic model of the temporal and azimuthal dispersion seen at the base station in outdoor propagation environments," IEEE Trans. Vehicular Technology, vol. 49, no. 2, pp. 437-447, 2000.

[30] A. Capone, M. Cesana, G. D'Onofrio, and L. Fratta, "Mixed traffic in UMTS downlink," IEEE Microwave and Wireless Components Letters, vol. 13, no. 8, pp. 299-301, 2003.

[31] J. Laiho, A. Wacker, and T. Novosad, Eds., Radio Network Planning and Optimisation for UMTS, John Wiley \& Sons, Chichester, UK, 2002.

[32] R. L. Cupo and R. D. Gitlin, "Adaptive carrier recovery systems for digital data communications receivers," IEEE Journal on Selected Areas in Communications, vol. 7, no. 9, pp. 13281339, 1989.

\section{Ramón Martínez Rodríguez-Osorio was} born in Madrid, Spain, in 1975. He received the Ingeniero de Telecomunicación degree in 1999 from the E.T.S.I. Telecomunicación, Universidad Politécnica de Madrid (UPM). In 1999, he joined the Departamento de Señales, Sistemas y Radiocomunicaciones at the same University, where he works as Assistant Professor and is currently working towards the Doctor Ingeniero de Telecomu-

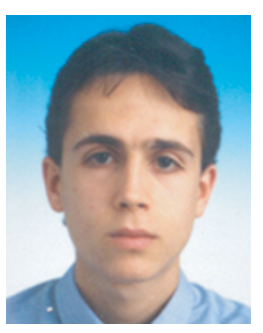
nicación (Ph.D.) degree. He participated in COST286 (European Project), working on the simulation of communication networks, and in other public and private funding research projects. He has also been involved in several other research projects related to DSP-based modems and smart antennas for UMTS, and in the development simulation platforms for mobile communications systems. He has also participated in field measurement campaigns in actual TV interference scenarios. His other research activities focus on the area of adaptive beamformer implementation for UMTS, and on the impulsive noise interference modelling and channel emulation, studying its impact on communications systems. He has published chapters in two books, and he has also contributed in a number of international conferences and journals.

Laura García García was born in Toledo, Spain, in 1978. She received the Ingeniero de Telecomunicación degree in 2001 from the E.T.S.I. Telecomunicación, Universidad Politécnica de Madrid (UPM). In that year, she joined the Departamento de Señales, Sistemas y Radiocomunicaciones at the same University, where she is currently working towards the Doctor Ingeniero de Telecomunicación (Ph.D.) degree. She has worked in several research projects related to smart antennas and their DSP-based implementation. Her other research interests are in the study and implementation of efficient algorithms for smart antennas, specially focused on DSP-based real-time systems.

Alberto Martínez Ollero was born in Madrid, Spain, in 1978. He received the Ingeniero de Telecomunicación degree from the E.T.S.I. Telecomunicación, Universidad Politécnica de Madrid (UPM) in 2002. In that year, he joined the Departamento de Señales, Sistemas y Radiocomunicaciones, UPM, where he is currently working in several research projects. His interests include the development of signal processing algo-

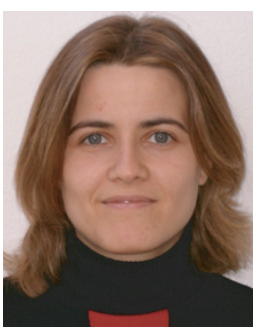
rithms for smart antennas and W-CDMA systems, implementation of software radio modules on DSP platforms for real-time applications and, antenna design for UMTS and DVB-S systems.

Francisco Javier García-Madrid Velázquez was born in Ciudad Real, Spain, on May 7, 1979. He received the Ingeniero de Telecomunicación degree from the Universidad Politécnica de Madrid (UPM) in 2002. In that year, he joined the Departamento de Señales, Sistemas y Radiocomunicaciones, UPM, where he is currently working toward the Ph.D. degree at the Escuela Técnica Superior de Ingenieros de Telecomunicación (E.T.S.I Telecomunicación). His research interests include the implementation of signal processing algorithms for smart antennas, studies of impulsive noise effects in communications systems, and fuzzy logic applied to channel estimation and beamforming.

Leandro de Haro Ariet received the Ingeniero de Telecomunicación degree in 1986 and the Doctor Ingeniero de Telecomunicación degree (Apto cum laude) in 1992, both from the E.T.S.I. Telecomunicación, Universidad Politécnica de Madrid (UPM) (Departamento de Señales, Sistemas y Radiocomunicaciones). Since 1990, he has developed his professional career in the Departamento de Señales, Sistemas y Radio-

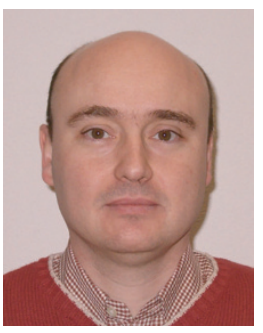
comunicaciones as Profesor Titular de Universidad in the signal 
theory and communications area. His research activity covers the following topics: antenna design for satellite communications (earth stations and satellite on board); study and design of satellite communication systems; and study and design of digital TV communication systems. He has been actively involved in several official projects and with private companies (national and international). He has also been involved in several European projects (RACE, ACTS, COST). The results of his research activity may be found in several presentations in national and international conferences as well as in published papers.

Miguel Calvo Ramón was born in Pueyo de Jaca, Huesca, Spain, on June 10, 1949. He received the Ingeniero de Telecomunicación degree from the E.T.S.I. Telecomunicación, Universidad Politécnica de Madrid (UPM) in 1974 and the Doctor Ingeniero de Telecomunicación degree $(\mathrm{PhD})$ from the same University in 1979. He works as a Catedrático (Full Professor) in the Departamento de Señales Sistemas y Radiocomuni-

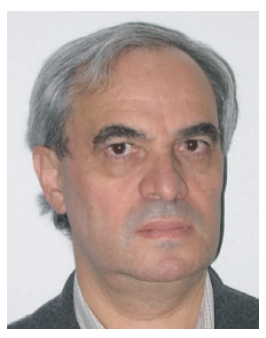
caciones since 1986. Since his incorporation to UPM in 1974, he has worked in a number of projects related to numerical methods in electromagnetics, EMC, antennas and testbed simulators for satellite communications (Hispasat). He has worked in the coordination procedures of the Spanish Hispasat satellite system with Intelsat, and part-time as a Technical Director of the Space Division at RYMSA. He has also worked as an Evaluator of Proposals in the framework of the European IST program. He was a Research Visitor at Queen Mary College, London University, in 1983 and Technical Visitor at Nichols Centre, Kansas University in Lawrence, in 1993. He has coauthored a number of papers in technical journals and contributed in a number of international conferences. He wrote a chapter in the book Reflector and Lens Antennas: Analysis and Design Using Personal Computers (C. J. Sletten, Ed., Artech House Publishers, 1988). 